\title{
Experimentally Verified Modeling of Erbium-Ytterbium Co-Doped DFB Fiber Lasers
}

\author{
Kuthan Yelen, Louise M. B. Hickey, and Mikhail N. Zervas, Member, IEEE
}

\begin{abstract}
For the first time, the simulation results of fiber distributed feedback (DFB) lasers are compared against experimental data in this paper. The pump source, active medium, and grating are all modeled and simulated to predict actual laser characteristics. Simple characterization methods are illustrated for the measurement of model parameters. Large loss at the pump wavelength is observed, attributed to the lifetime quenching of $\mathbf{Y b}$ ions, and included in the model as a critical parameter. DFB lasers with two different apodization profiles successfully simulated with the same set of model parameters.
\end{abstract}

Index Terms-Distributed feedback (DFB) lasers, erbium, optical fiber lasers, optical fiber theory, ytterbium.

\section{INTRODUCTION}

$\mathbf{E}$ RBIUM-YTTERBIUM-co-doped fiber distributed feedback (DFB) lasers are important devices, finding applications in many areas ranging from telecom [1]-[3] to sensors [4]-[6] due to their inherit fiber compatibility, stable single longitudinal mode, and single polarization operation. In addition, their emitted signal wavelength can be set accurately with passive stabilization. They have low phase noise as well as low relative intensity noise (RIN) and narrow linewidth [7]. A number of DFB fiber lasers can be configured in a parallel array to provide flexibility in pumping conditions and pump redundancy [8]. An accurate model of these types of lasers is very desirable since the simulations can reduce the design and development time and cost significantly. A model can also help to improve the physical insight on the device or system under investigation by allowing one to carry out calculations and hypothetical experiments, which in many cases can be extremely difficult in laboratory conditions.

There are two major difficulties in accurate modeling of $\mathrm{Er}-\mathrm{Yb}$ co-doped devices. The first difficulty is the large number of equations that have to be solved: when pumped around $980 \mathrm{~nm}$ and operating around $1550 \mathrm{~nm}$, an Er-Yb-co-doped medium allows multiple interactions between ions and propagating fields [9]. In addition to the basic absorption and

Manuscript received July 28, 2004; revised November 172004.

$\mathrm{K}$. Yelen is with the Optoelectronics Research Centre (ORC), University of Southampton, Southampton SO17 1BJ, U.K. (e-mail: ky@orc.soton.ac.uk).

L. M. B. Hickey is with SPI (Southampton Photonics, Inc.), Southampton SO30 2QU, U.K. (e-mail: louise.hickey@ spioptics.com).

M. N. Zervas is with the Optoelectronics Research Centre (ORC), University of Southampton, Southampton SO17 1BJ, U.K., and also with SPI (Southampton Photonics, Inc.), Southampton SO30 2QU, U.K. (e-mail: mikhail.zervas@spioptics.com).

Digital Object Identifier 10.1109/JLT.2005.843477 emission transitions of a quasi-three-level system, there is energy transfer between $\mathrm{Er}$ and $\mathrm{Yb}$ ions, in both directions; the excited state absorption (ESA) of both pump and signal is possible; cooperative upconversion for ions at various excited states is allowed, which may be a two-, three-, or many-ion interaction; and the relaxation of excited ions can be through phonon emission as well as spontaneous emission, such as at green light wavelengths. Also lifetime quenching of $\mathrm{Yb}$ ions has been reported [10]. Therefore, the full description of the medium would include many linear and nonlinear equations. The second difficulty is the measurement of the actual values of the parameters and coefficients that appear in these equations. The measurement of the active medium properties requires detailed spectroscopic investigation [11], [12], and data usually represent the combined effect of various parameters. Therefore, certain assumptions and fitting of some parameters cannot be avoided.

The solution of the ideal model with all the possible transitions is impractical. Therefore, in practice, the solution of complex active medium equations, whether it is an analytical or numerical solution, inevitably requires simplifications and approximations. Ignoring some of less significant transitions, a set of equations reported to be successfully modeling the $\mathrm{Er}-\mathrm{Yb}-$ doped medium in the amplifier regime [13] and purely theoretical works investigating the implications of these simplifications for DFB laser modeling have been reported [14], [15]. In theoretical works, however, it is critical that one addresses the actual values of the ignored transitions. Assuming a small coefficient for an interaction and then simulating the media with and without this interaction obviously would not result in a significant variation as in the case of [15]. In reality, however, if the coefficient is large, then the transition must be included for accurate modeling. Therefore, the simulation results must be compared against experimental data in order to validate the assumptions so that anything beyond trends can be concluded about the device performance by using the simulations.

Although several entirely theoretical work on modeling of erbium-ytterbium-co-doped media have been reported [9], [14]-[19] with only very few experimental verification for amplifiers [13], [20], there is no work benchmarking the simulation results with experimental results for $\mathrm{Er}-\mathrm{Yb}$-co-doped lasers, whether in Fabry-Pérot, distributed Bragg reflector (DBR), or DFB configuration. This paper presents a model for Er-Yb-co-doped fiber DFB lasers with a minimum number of critical transitions, which, for the first time, predict actual characteristics of the Er-Yb-co-doped DFB fiber lasers within the 


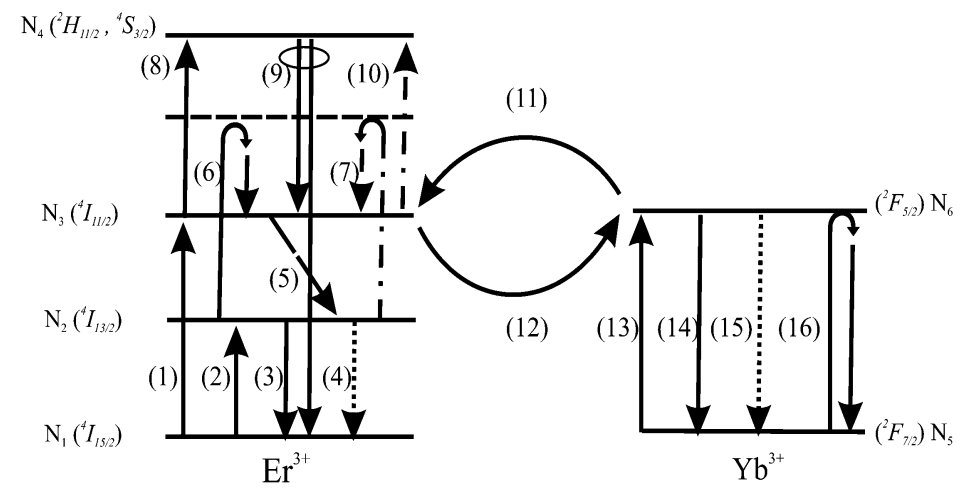

Fig. 1. Energy scheme of Er and $\mathrm{Yb}$ ions. Arrows with dashees and dots indicate cooperative upconversion; dotted lines are used for spontaneous emission.

TABLE I

DESCRIPTION OF TRANSITIONS IN FIG. 1

\begin{tabular}{|c|c|c|}
\hline$\#$ & Transition Type & Transition Detail \\
\hline 1 & Absorption (Pump) & $\mathrm{Er}_{1}+\lambda_{\mathrm{p}} \rightarrow \mathrm{Er} 3$ \\
\hline 2 & Abs orption (Signal) & $\operatorname{Er}_{1}+\lambda_{s} \rightarrow \operatorname{Er} 2$ \\
\hline 3 & Stimulated Emission (Signal) & $\mathrm{Er}_{2}+\lambda_{\mathrm{s}} \rightarrow \mathrm{Er}_{1}+2 \lambda_{\mathrm{s}}$ \\
\hline 4 & Spontaneous Emission & $\operatorname{Er}_{2} \rightarrow \operatorname{Er}_{1}+\lambda$ \\
\hline 5 & Non-radiative Decay & $\mathrm{Er}_{3} \rightarrow \mathrm{Er}_{2}+$ phonon \\
\hline 6 & ESA (Signal) and Fast Decay & $\mathbf{E r} 2+\lambda_{s} \rightarrow \mathbf{E r} 3+$ phonon \\
\hline 7 & CUC and Fast Decay & $\mathrm{Er}_{2}+\mathrm{Er}_{2} \rightarrow \mathrm{Er}_{1}+\mathrm{Er}_{3}+$ phonon \\
\hline 8 & ESA (Pump) & $\mathrm{Er}_{3}+\lambda_{\mathrm{p}} \rightarrow \mathrm{Er}_{4}$ \\
\hline 9 & Radiative and Non-radiative Decay & $\mathrm{Er}_{4} \rightarrow \mathrm{Er}_{3}+\lambda_{\text {green }}+\mathrm{Er}_{1}+$ phonon \\
\hline 10 & CUC & $\mathbf{E r}_{3}+\mathrm{Er}_{3} \rightarrow \mathrm{Er}_{1}+\mathrm{Er}_{4}$ \\
\hline 11 & Yb to Er Energy Transfer (Forward) & $\mathrm{Yb}_{6}+\mathrm{Er}_{1} \rightarrow \mathrm{Yb}_{5}+\mathrm{Er}_{3}$ \\
\hline 12 & Er to Yb Energy Trans fer (Backward) & $\mathrm{Er}_{3}+\mathrm{Yb}_{5} \rightarrow \mathrm{Yb}_{6}+\mathrm{Er}_{1}$ \\
\hline 13 & Absorption (Pump) & $\mathrm{Yb}_{5}+\lambda_{\mathrm{p}} \rightarrow \mathrm{Yb}_{6}$ \\
\hline 14 & Stimulated Emission (Pump) & $Y_{b_{6}}+\lambda_{p} \rightarrow Y_{b s}+2 \lambda_{p}$ \\
\hline 15 & Spontaneous Emission & $\mathrm{Yb}_{6} \rightarrow \mathrm{Yb}_{5}+\lambda$ \\
\hline 16 & Abs orp. \& Nonrad. Trans. In Quenched Ions & Ybs $+\lambda_{p} \rightarrow$ Ybs + phonon \\
\hline
\end{tabular}

range of experimental measurement errors. Simple techniques to measure and deduce the required parameters are described. The pump, the active medium, and the grating are characterized individually. In addition to the traditional parameters, the lifetime quenching of $\mathrm{Yb}$ ions are identified as a key parameter in the efficiency of Er-Yb-doped fiber lasers.

\section{Characterization of Active Medium}

\section{A. Erbium-Ytterbium Model}

The Er-Yb-co-doped medium is described by a set of rate equations derived from the transitions between energy levels due to ion-ion and ion-light interactions. Ion-ion interactions we consider are cooperative upconversion among Er ions and energy transfer between $\mathrm{Er}$ and $\mathrm{Yb}$ ions. In both cases, we assume that a two-ion interaction takes place, although more ions exchanging energy at the same time is also possible. Ion-light interactions include absorption at the ground state, stimulated emission, and absorption at an ESA. In addition to these transitions, we also consider spontaneous emission and nonradiative transitions.

In principle, any energy transfer between ions and propagating fields is possible as long as there is an energy band that can accommodate the ions. Here, we focused only on the transitions that have been reported to have observable effects. Fig. 1 shows these transitions, and Table I details the mechanisms behind them. In addition, in reality, the cross sections vary with temperature [21], [22]. In our model, we neglect this effect for the sake of simplicity. Here, $N_{1}$ is the concentration of the Er ions at the ${ }^{4} \mathrm{I}_{15 / 2}$ level. An ion at this energy level is indicated by $\mathrm{Er}_{1}$ in Table I. Similarly $N_{2}$ stands for the concentration at ${ }^{4} \mathrm{I}_{13 / 2}, \mathrm{~N}_{3}{ }^{4} \mathrm{I}_{11 / 2}, \mathrm{~N}_{4}{ }^{2} \mathrm{H}_{11 / 2}$, and ${ }^{4} \mathrm{~S}_{3 / 2}$ together. For $\mathrm{Yb}$ ions, $N_{5}$ is the concentration at ${ }^{2} \mathrm{~F}_{7 / 2}$ and $\mathrm{N}_{6}$ at ${ }^{2} \mathrm{~F}_{5 / 2}$. Again, $\mathrm{Er}_{2}$, $\mathrm{Er}_{3}, \mathrm{Er}_{4}, \mathrm{Yb}_{5}$, and $\mathrm{Yb}_{6}$ stand for a single ion at the corresponding level. In our model, we neglect the time Er ions spend at the ${ }^{4} I_{9 / 2}$ level indicated with the horizontal dashed line; therefore, it does not appear in the rate equations.

In Table I, a photon emitted due to spontaneous emission at a wavelength different than pump $\left(\lambda_{p}\right)$ or signal $\left(\lambda_{s}\right)$ is shown 


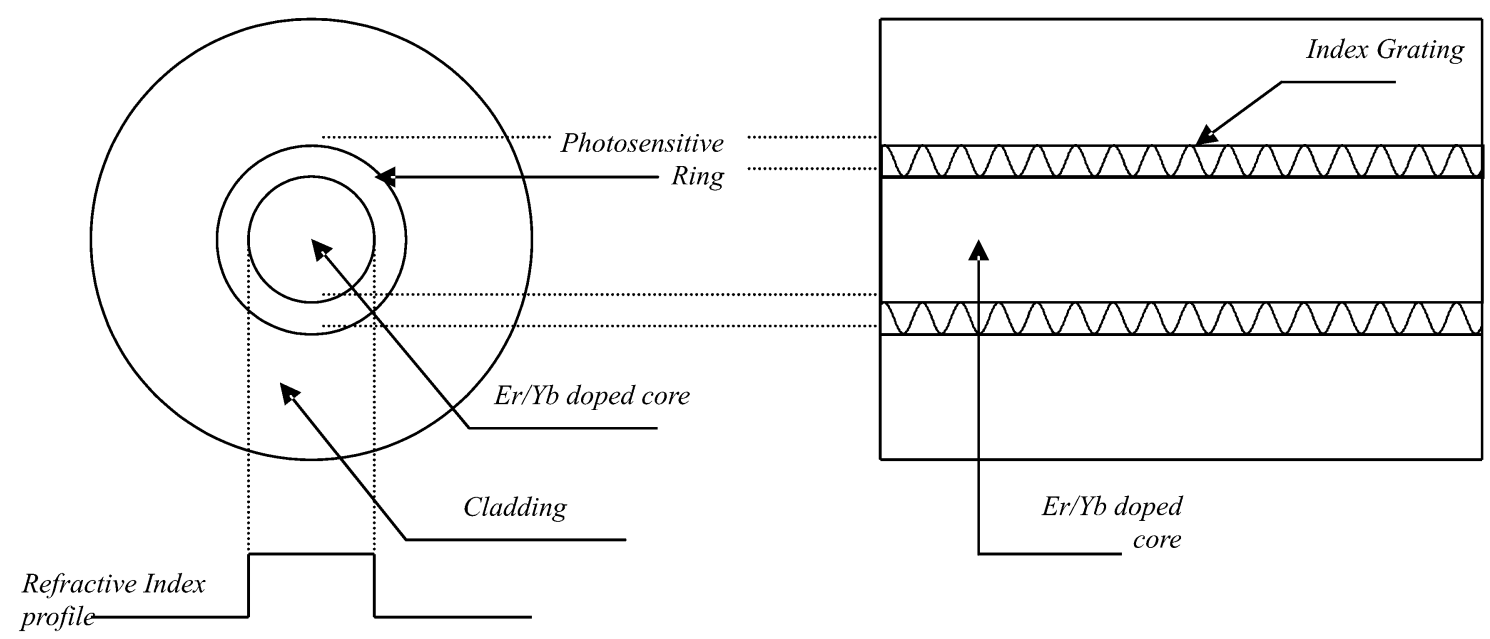

Fig. 2. Structure of the Er-Yb-doped photosensitive fiber. Refractive index is assumed to have a step profile. Grating is written in the photosensitive.

with $\lambda$, whereas $\lambda_{\text {green }}$ indicates a photon emitted between 514 and $532 \mathrm{~nm}$ in the green-light range, which was observed during experiments with this particular fiber when 980-nm pumping was used.

These transitions result in the following rate equations that should be equal to zero at the steady state:

$$
\begin{aligned}
\frac{d N_{1}}{d t}= & N_{2} R_{21}+N_{4} R_{41}+k_{\mathrm{tr}} N_{3} N_{5, n} \\
& +C_{\mathrm{UP}, 2} N_{2}^{2}+C_{\mathrm{UP}, 3} N_{3}^{2}-N_{1} R_{13} \\
& -N_{1} R_{12}-k_{\mathrm{tr}} N_{6, n} N_{1}=0 \\
\frac{d N_{2}}{d t}= & N_{3} R_{32}+N_{1} R_{12}-N_{2} R_{21} \\
& -2 C_{\mathrm{UP}, 2} N_{2}^{2}-C_{\mathrm{ESA}, 2} \frac{I_{s}}{h \nu_{s}} N_{2}=0 \\
\frac{d N_{3}}{d t}= & N_{1} R_{13}+k_{\mathrm{tr}} N_{6, n} N_{1}+C_{\mathrm{ESA}, 2} \frac{I_{s}}{h \nu_{s}} N_{2} \\
& +C_{\mathrm{UP}, 2} N_{2}^{2}+N_{4} R_{43}-N_{3} R_{32}-N_{3} R_{31} \\
& -k_{\mathrm{tr}} N_{5, n} N_{3}-2 C_{\mathrm{UP}, 3} N_{3}^{2}-C_{\mathrm{ESA}, 3} \frac{I_{p}}{h \nu_{p}} N_{3}=0
\end{aligned}
$$

$$
\begin{aligned}
\frac{d N_{4}}{d t}= & C_{\mathrm{UP}, 3} N_{3}^{2}+C_{\mathrm{ESA}, 3} \frac{I_{p}}{h \nu_{p}} N_{3} \\
& -N_{4} R_{41}-N_{4} R_{43}=0 \\
\frac{d N_{5, n}}{d t}= & N_{6, n} R_{65}+k_{\mathrm{tr}} N_{6, n} N_{1} \\
& -N_{5, n} R_{56}-k_{\mathrm{tr}} N_{3} N_{5, n}=0 \\
\frac{d N_{5, q}}{d t}= & N_{6, q} R_{q}-N_{5, q} R_{56} \\
& N_{6, n}=N_{n}^{\mathrm{Yb}}-N_{5, n} \quad N_{6, q}=N_{q}^{\mathrm{Yb}}-N_{5, q} \\
& N_{1}+N_{2}+N_{3}+N_{4}=N^{\mathrm{Er}} \\
& N_{5, n}+N_{6, n}+N_{5, q}+N_{6, q}=N^{\mathrm{Yb}} .
\end{aligned}
$$

Here, $R_{x y}$ stands for the transition rate from level $x$ to level $y$, and individual definitions of these rates are as follows:

$$
R_{12}=\frac{I_{s}}{h \nu_{s}} \sigma_{s, a}^{\mathrm{Er}}, \quad R_{13}=\frac{I_{p}}{h \nu_{p}} \sigma_{p, a}^{\mathrm{Er}}
$$

$$
\begin{aligned}
R_{21} & =\frac{I_{s}}{h \nu_{s}} \sigma_{s, e}^{\mathrm{Er}}+\frac{1}{\tau_{2}} \\
R_{31} & =\frac{I_{p}}{h \nu_{p}} \sigma_{p, e}^{\mathrm{Er}}, \quad R_{32}=\frac{1}{\tau_{3}} \\
R_{56, n} & =\frac{I_{p}}{h \nu_{p}} \sigma_{s, a}^{\mathrm{Yb}}, \quad R_{65, n}=\frac{I_{p}}{h \nu_{p}} \sigma_{p, e}^{\mathrm{Yb}}+\frac{1}{\tau_{6, \text { normal }}} \\
R_{56, q} & =\frac{I_{p}}{h \nu_{p}} \sigma_{s, a}^{\mathrm{Yb}}, \quad R_{q}=\frac{I_{p}}{h \nu_{p}} \sigma_{p, e}^{\mathrm{Yb}}+\frac{1}{\tau_{6, \text { quenched }}} .
\end{aligned}
$$

In the rate equations for $\mathrm{Yb}$ ions, subscript $n$ stands for the normal ions, and $q$ denotes the lifetime-quenched ions. The need to separate the $\mathrm{Yb}$ concentration in normal and quenched subgroups will be justified after the full spectroscopy and absorption at the pump wavelength is considered subsequently in Section C. Above, $I$ is the intensity, $\sigma$ is the cross section, $\nu$ is the frequency, $\tau$ is the lifetime, subscripts $a$ and $e$ are absorption and emission, $p$ and $s$ denote pump and signal, respectively, and numbers follow the numbering of levels in Fig. 1. $C_{\mathrm{ESA}, x}$ and $C_{\mathrm{UP}, x}$ are ESA cross-section and upconversion rates from level $x$, respectively. For radiative green-light transition, $R_{41}$ from level 4 and nonradiative transition $R_{43}$, we use the average constant values $10^{3} \mathrm{~s}^{-1}$ and $14 \times 10^{5} \mathrm{~s}^{-1}$, respectively [23].

Equations (1)-(8) can be solved numerically to provide the steady-state values of the population concentrations for given signal and pump intensities. In our implementation, we assume a value for $N_{3}$ and substitute this in (4) to solve for $N_{4}$. Then, (2) and (7) are used to solve for $N_{1}$ and $N_{2}$. Finally, (5), (6), and (8) are used to find $N_{5, n}, N_{6, n}, N_{5, q}$, and $N_{6, q}$. We check the validity of our initial assumption for $N_{3}$ by substituting $N_{1}, N_{2}, N_{3}, N_{4}, N_{5, n}$, and $N_{6, n}$ in (1); if the assumption is correct, then the result should be zero. We vary the $N_{3}$ value until (1) is smaller than a tolerance value.

\section{B. Fiber Composition and Geometry}

The fiber is produced using the modified chemical vapor deposition (MCVD) technique [24]. The core of the fiber is a phosphosilicate glass doped with $\mathrm{Er}, \mathrm{Yb}$, and aluminum. This active core is surrounded by a photosensitive germanosilicate ring doped with boron that matches the refractive index of the silica cladding. Fig. 2 illustrates the geometrical properties of the fiber. 
The core radius of the fiber is $2.3 \mu \mathrm{m}$, and the cutoff wavelength is $1150 \mathrm{~nm}$, giving NA $=0.1921$. The refractive index of cladding $\left(n_{2}\right)$ is 1.4585 , and we calculate the core index $\left(n_{1}\right)$ to be 1.4709 . In our simulations, we assume the refractive-index distribution to have a step profile across the cross section of the fiber. We also assume the dopands to be uniformly distributed in the core. The refractive-index grating is written in the photosensitive ring by exposing the fiber to an ultraviolet (UV) interference pattern produced by a phase mask [25], [26].

\section{Spectroscopy at Pump Wavelength}

In the absence of a grating, if a signal with power $P_{S}$ and pump with power $P_{P}$ propagate in an active medium in $+z$ direction, then the propagation equations are of the form

$$
\frac{d P_{s}}{d z}=2 \alpha_{s}(z) P_{s}(z), \quad \frac{d P_{p}}{d z}=-2 \alpha_{p}(z) P_{p}(z)
$$

where $\alpha_{s}$ and $\alpha_{p}$ are the gain and the absorption coefficients for the signal and pump fields, reflectively.

The fiber DFB lasers we investigate are pumped at around 980 $\mathrm{nm}$. Around this wavelength, the $\mathrm{Yb}$ absorption is largest, which leads to the maximum energy transfer from pump to signal over the shortest length. The total pump absorption coefficient $2 \alpha_{p}$ by both $\mathrm{Yb}$ and Er ions is given by

$$
\begin{aligned}
2 \alpha_{P}=( & N_{5} \sigma_{p, a}^{\mathrm{Yb}}-N_{6} \sigma_{p, e}^{\mathrm{Yb}}+N_{1} \sigma_{p, a}^{\mathrm{Er}} \\
& \left.-N_{3} \sigma_{p, e}^{\mathrm{Er}}+N_{3} C_{\mathrm{ESA}, 3}\right) \Gamma_{P}+2 \alpha_{P, \text { background }}
\end{aligned}
$$

where $\Gamma_{p}$ is the overlap coefficient between the pump power distribution and the active medium cross section. $\alpha_{p}$,background stands for any constant background loss at the pump wavelength. If the pump power is very small, then at steady state only a small fraction of the ions will be in their excited energy levels. In (10), the number of excited $\mathrm{Yb}$ ions is denoted with $N_{6}$, and the number of excited Er ions is $N_{3}$. If we neglect these terms for small pump powers, when practically all the ions are in their ground state, then the absorption coefficient can be approximated with

$$
2 \alpha_{p} \approx\left(N^{\mathrm{Yb}} \sigma_{p, a}^{\mathrm{Yb}}+N^{\mathrm{Er}} \sigma_{p, a}^{\mathrm{Er}}\right) \Gamma_{p}
$$

assuming the background loss is small compared with the absorption by the ions. Around $980 \mathrm{~nm}$, the $\mathrm{Yb}$ absorption cross section is significantly larger than the Er absorption cross section. In addition to this, in the fiber, we investigate a larger concentration of $\mathrm{Yb}$ was used compared with $\mathrm{Er}$ in order to reduce quenching of Er ions [12] and to optimize the energy transfer. From the production of the fiber, we know that the concentration of $\mathrm{Yb}$ will be more than ten times larger compared with the Er concentration. Therefore, we can further simplify the absorption coefficient to

$$
2 \alpha_{p} \approx N^{\mathrm{Yb}} \sigma_{p, a}^{\mathrm{Yb}} \Gamma_{p}
$$

We applied the cutback method in order to measure the absorption coefficient $2 \alpha_{p}$ of the fiber at low pump powers. We used a tunable semiconductor laser as the pump source and measured its output power as $-55 \mathrm{dBm}$ over the range of 950 to

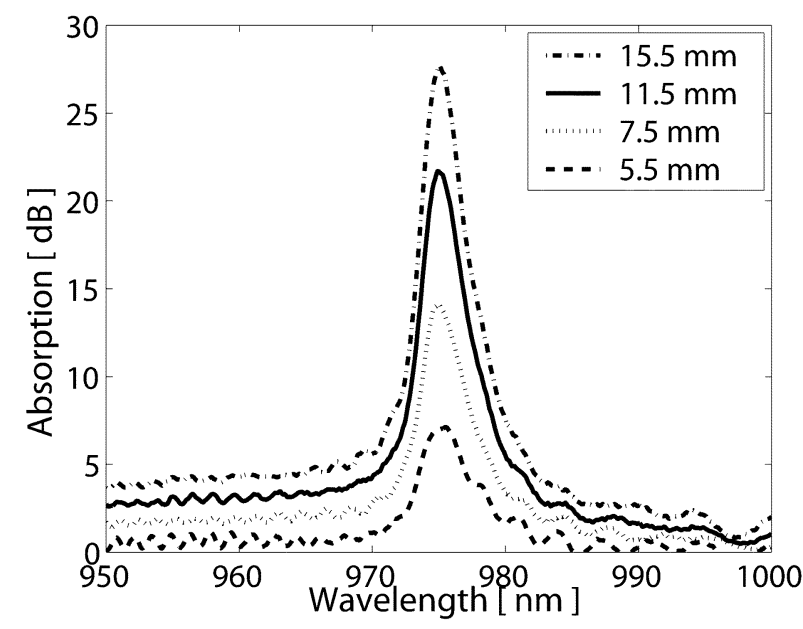

Fig. 3. Pump absorption in the Er-Yb co-doped fibers of different length.

$1000 \mathrm{~nm}$ by directly connecting the laser to a photodetector. We then spliced a 15.5-mm-long Er-Yb co-doped fiber between the pump source and the detector and measured the transmitted power. We remeasured the transmitted power after removing 4-, 8-, and 10-mm-long pieces of fiber. We calculated the pump absorption in each case by comparing the transmitted power when the fiber was present with the power measured without the fiber. Fig. 3 shows the results of this experiment after an estimated 0.5-dB splice loss was subtracted.

By comparing the absorption by different length of fiber segments with each other, we obtained an average value of the absorption per unit length. Although the pump power is delivered to the fiber in single mode, the Er-Yb-co-doped fiber has a varying $V$ value from 2.89 to 2.74 for wavelengths 950-1000 nm; therefore, the pump power is divided into two modes. Since the fiber lengths we used were short, we estimated $90 \%$ of the pump power to remain in the fundamental mode and $10 \%$ to be in the higher mode. The wavelength-dependent $V$ parameter leads to wavelength-dependent Bessel's functions describing the distribution of the modes across the cross section of the fiber. For each wavelength, we numerically integrate these equations in order to find the overlap between the fields and the active core assuming step-index refractive-index profile and uniformly distributed dopants. Calculating the wavelength-dependent overlap coefficient $\Gamma_{p}(\lambda)$ for pump fields and substituting the measured absorption coefficient in (11), we calculated the $N^{\mathrm{Yb}} \sigma_{p, a}^{\mathrm{Yb}}(\lambda)$ term (see Fig. 4).

The small-power absorption coefficient given in Fig. 4 is a result of the combined effect of the total concentration and the absorption cross section of $\mathrm{Yb}$ ions; therefore one needs to choose either one of them as the fitting parameter, and the other one will be derived from (11). We will use total $\mathrm{Yb}$ concentration, $N^{\mathrm{Yb}}$, as the fitting parameter and derive the absorption cross section, so that we can justify our assumption by comparing the resulting spectra with the previously reported values.

If the parameters are accurate enough, then the model should also be able to predict high-power absorption characteristics. Therefore, we also measured pump absorption of the fiber at high powers. We used a semiconductor laser of wavelength 979 $\mathrm{nm}$ whose output power could be varied from 0 to $240 \mathrm{~mW}$. We 


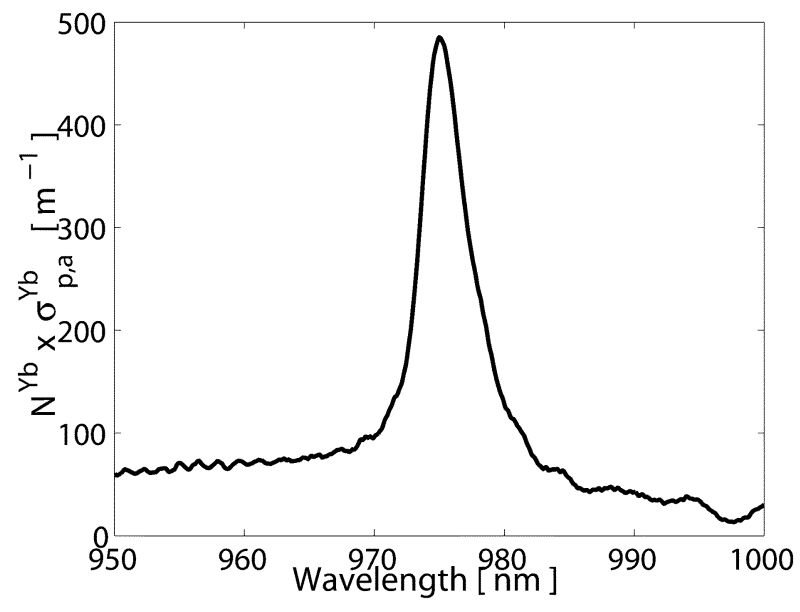

Fig. 4. Average pump absorption per unit length.

again applied the cutback method in order to calculate absorption per unit length of the fiber at high powers. By comparing the pump power before and after a segment, we were able to calculate the pump-power-dependent absorption of the segment. Since the removed segment lengths were short, we assumed the pump power had a constant average value along the removed fiber segment.

Fig. 5 shows the variation of absorption per unit length with the increasing pump power. Each mark in the figure gives the absorption calculated by comparing the transmitted power by two different fiber lengths. The $(+)$ is obtained by comparing the transmitted powers at the end of 25- and 35-mm-long fibers; $(x)$ compares 25 - and $40-\mathrm{mm}$-long fibers, and $(*)$ compares 35 and 40-mm-long fibers.

As the pump power increases, absorption decreases due to saturation of the medium: with increasing pump power, more of the $\mathrm{Yb}$ ions move to the excited state; however, there are only a finite number of ions. Therefore, in the ground state, fewer ions remain for further absorption and, consequently, the absorption drops.

However, the results of this experiment at the high-power end show a nontypical saturation behavior. Although saturation is clearly observed, absorption does not drop to very small values, which should be the case if only a small background loss is present in the system. In the present case the absorption, $2 \alpha_{p}$ becomes asymptotic to a significantly large value around $20 \mathrm{~m}$ ${ }^{-1}(87 \mathrm{~dB} / \mathrm{m})$.

This value is too large to be a constant background loss due to fiber impurities or splicing losses. This loss may be the result of the intrinsic $\mathrm{Er}$ or $\mathrm{Yb}$ transition dynamics in the medium. If this is the case, we expect it to be related to $\mathrm{Yb}$ ions since the concentration as well as the absorption cross section of $\mathrm{Yb}$ ions at this particular wavelength are significantly larger than that of Er ions. In order to check this assumption, we measured the saturation characteristics of only $\mathrm{Yb}$-doped fibers produced by same method using the same facilities and we observed similar significant unsaturable loss values at large power, as shown in Fig. 6.

Fig. 6 shows that the larger the $\mathrm{Yb}$ concentration, the larger the amount of unsaturable loss. The fiber with lower $\mathrm{Yb}$ concentration $\left(8.5 \times 10^{25} \mathrm{~m}^{-3}\right)$ exhibited an unsaturable loss 2

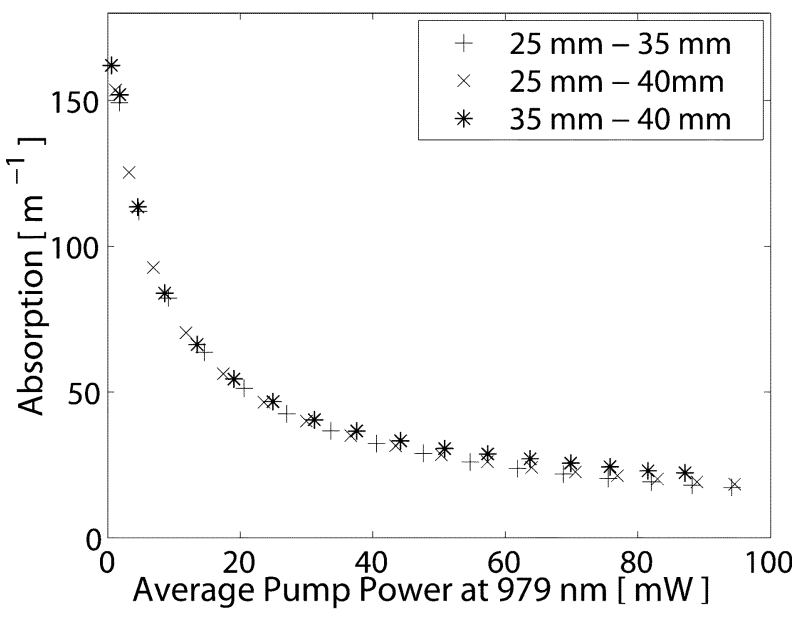

Fig. 5. Variation of absorption with pump power. The data were obtained by comparing the pump powers leaving different lengths of fibers $(25-35 \mathrm{~mm}$, $25-40 \mathrm{~mm}, 35-40 \mathrm{~mm}$ ). Figure indicates the existence of an unsaturable loss around $20 \mathrm{~m}^{-1}$.

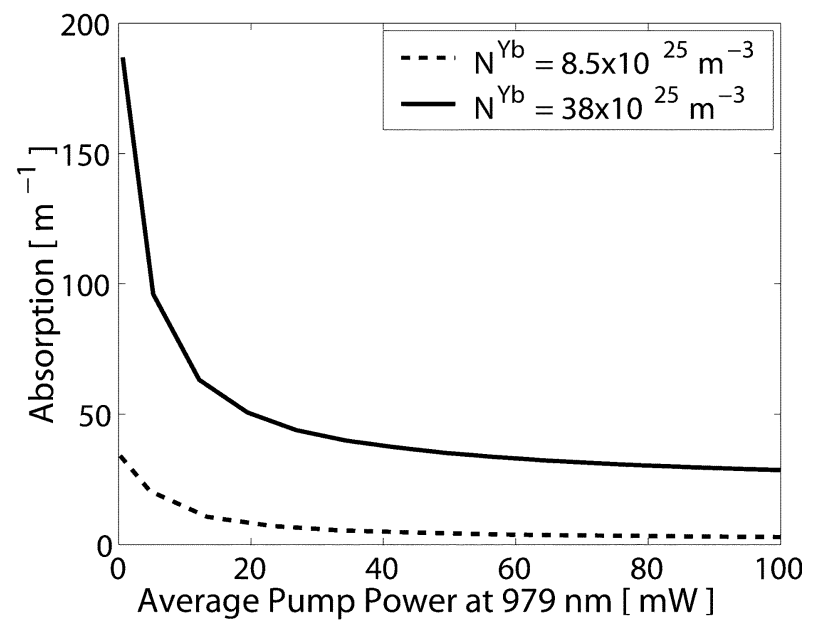

Fig. 6. Absorption saturation characteristics of Yb-only-doped fibers, produced in the same way as the Er-Yb-doped fiber. Both fibers exhibit an unsaturable loss.

$\alpha_{\text {unsaturable }}=3 \mathrm{~m}^{-1}(13 \mathrm{~dB} / \mathrm{m})$, whereas in the fiber with large $\mathrm{Yb}$ concentration $\left(38 \times 10^{25} \mathrm{~m}^{-3}\right)$, this value was as large as $30 \mathrm{~m}^{-1}(130 \mathrm{~dB} / \mathrm{m})$. The unsaturable loss phenomenon in Yb-only-doped media has been reported previously [10], [27], [28]. Although the exact mechanism behind this absorption has not yet been explained, the overall effect appears to be due to lifetime quenching of a portion of $\mathrm{Yb}$ ions. An extremely small lifetime causes the excited state ions to return to ground state very quickly through some nonradiative relaxation. Since this cycle is completed very fast, absorption due to the quenched ions appears to be an unsaturable loss in addition to normal $\mathrm{Yb}$ saturable absorption [10].

In modeling accurately the performance of Er-Yb-codoped DFB lasers, we tried to account for the observed unsaturable loss by considering the excited state absorption and the cooperative upconversion in Er ions. In order to get reasonable agreement with the experimental data, the required parameters describing 


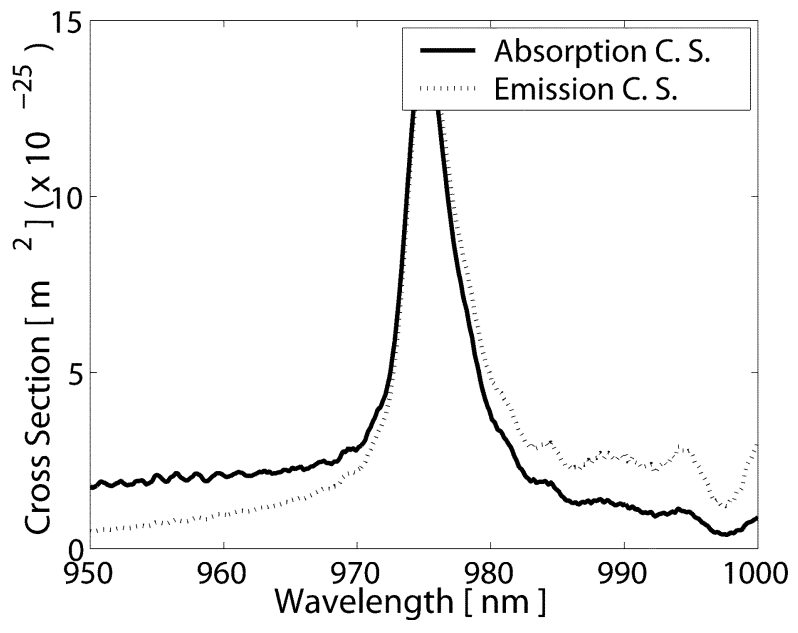

Fig. 7. Calculated absorption (solid line) and emission (dotted line) cross sections of $\mathrm{Yb}$ ions. The absorption cross section is calculated from the experimental absorption spectrum, and the emission cross section is derived using McCumber theory.

these secondary transitions had to be well out of the range reported in the literature. Therefore, we conclude that the unsaturable loss in the Er-Yb-doped fiber around $980 \mathrm{~nm}$ was related to the lifetime quenching of $\mathrm{Yb}$ ions. If we assume that the lifetime-quenched $\mathrm{Yb}$ ions are practically always in the ground state, then we can write

$$
2 \alpha_{\text {unsaturable }} \approx N_{\text {quenched }}^{\mathrm{Yb}} \sigma_{p, a}^{\mathrm{Yb}} \Gamma_{p} .
$$

When we set the total $\mathrm{Yb}$ concentration, (11) and experimental data, shown in Fig. 4, give the absorption cross section $\sigma_{p, a}^{\mathrm{Yb}}$. Substituting this value in (12), we can calculate the concentration of quenched ions $N_{\text {quenched. }}^{\mathrm{Yb}}$. In doing so, we assume that normal and quenched $\mathrm{Yb}$ ions have the same emission and absorption cross sections and only differ in their upper laser level lifetimes.

For the Er-Yb-co-doped fiber, we achieved the best agreement between simulation and experimental results when $N^{\mathrm{Yb}}=$ $34 \times 10^{25} \mathrm{~m}^{-3}$. The low-pump-power experiment then gives $\sigma_{p, a}^{\mathrm{Yb}}(979 \mathrm{~nm})=5.89 \times 10^{-25} \mathrm{~m}^{-2}$, and $22 \mathrm{~m}^{-1}$ unsaturable loss requires $11 \%$ of total $\mathrm{Yb}$ ions to be quenched. We again use (11) and the data in Fig. 4 in order to calculate the absorption cross-section spectrum of the $\mathrm{Yb}$ ions. The results are shown in Fig. 7 with the solid curve.

Fig. 7 also shows the emission cross section of $\mathrm{Yb}$ ions calculated by using McCumber theory (e.g., see [29]), assuming emission and absorption cross sections are equal at $975 \mathrm{~nm}$ [30]. Both cross-sections spectra are within the range of values reported previously [30]-[32]

\section{Spectroscopy at Signal Wavelength}

In the Er-Yb-co-doped media, the signal is generated by the Er ions in the excited state undergoing radiative transitions. Using a low-power white-light source, we measured the absorption spectra of the fibers between 1400 and $1600 \mathrm{~nm}$, a range over which $\mathrm{Yb}$ ions are transparent. At low powers, we can assume all the Er ions to be in the ground state; therefore,
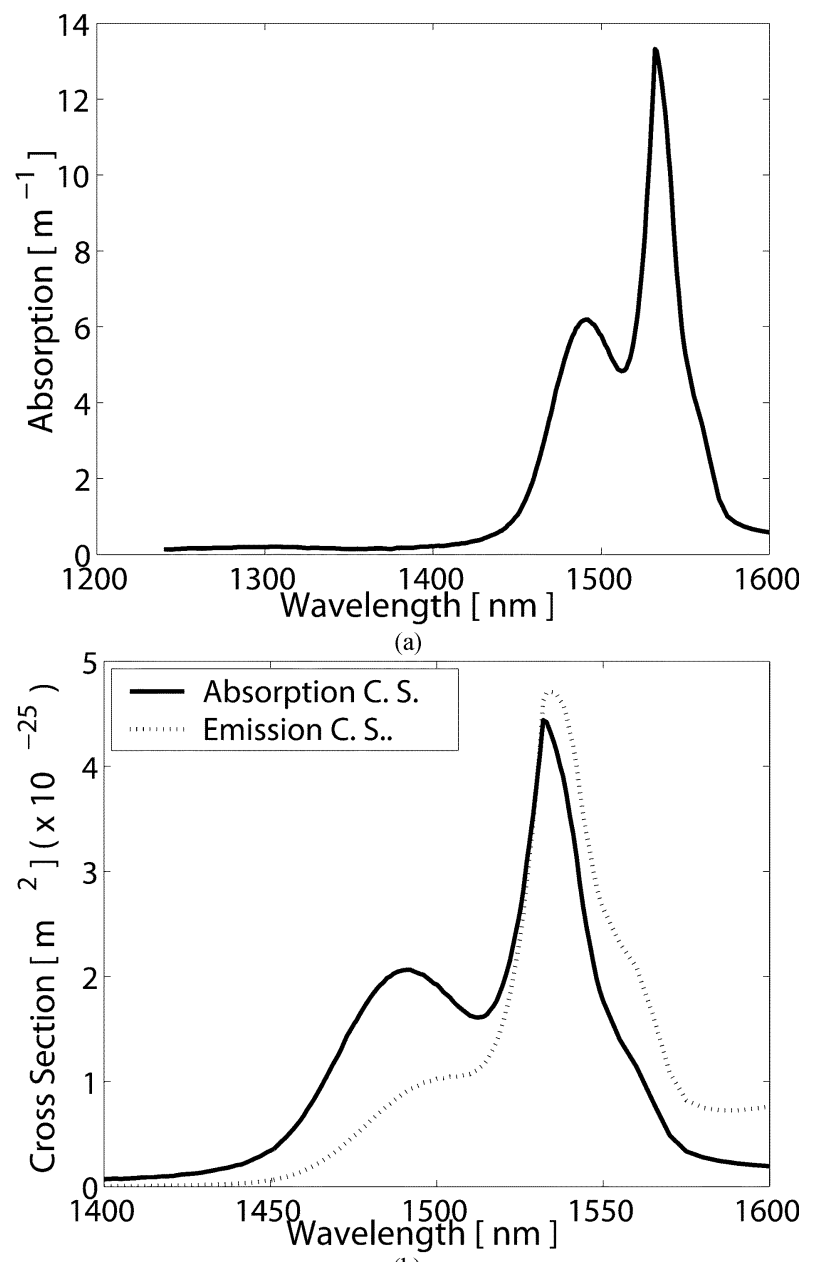

(b)

Fig. 8. (a) Absorption spectrum of Er ions, measured using a white-light source and employing cutback method. Absorption value at wavelengths lower then $1400 \mathrm{~nm}$ indicates a background loss of $0.15 \mathrm{~m}^{-1}$, since at these wavelengths both Er and $\mathrm{Yb}$ ions are transparent. (b) Absorption (solid line) and emission (dotted line) cross section. Emission spectrum is calculated using McCumber theory.

we can neglect any emission or excited state absorption or any upconversion from excited states. In this case, absorption of signal $2 \alpha_{s}$ can be used to determine the total ion concentration times the absorption cross section, as follows:

$$
N^{\mathrm{Er}} \sigma_{s, a}^{\mathrm{Er}} \approx \frac{2 \alpha_{s}}{\Gamma_{s}}
$$

where $\Gamma_{s}$ is the overlap between the active medium and the signal power distribution at the considered wavelength.

First, we measured the white-light source's output spectrum without any fiber attached to obtain the calibration data. Then, by introducing the fiber between the source and the detector and by gradually removing small lengths, we applied the cutback method to find the absorption per unit length as shown in Fig. 8(a). Calculating $\Gamma_{s}(\lambda)$, and assuming Er concentration to be $N^{\mathrm{Er}}=3 \times 10^{25} \mathrm{~m}^{-3}$, (13) gives the absorption cross-section spectrum of Er ions as shown in Fig. 8(b) with the solid line.

The absorption outside the absorption spectrum of both Er and $\mathrm{Yb}$ ions (between 1250 and $1400 \mathrm{~nm}$ ) is the constant background loss due to the fiber impurities and splicing losses. We 
TABLE II

ACTIVE MEDIUM MODEL PARAMETERS

\begin{tabular}{|c|c|c|c|}
\hline Parameter & Value & Source & Related Literature \\
\hline Pump Wavelength & $978-980 \mathrm{~nm}$ & Measured & \\
\hline $\begin{array}{l}\mathrm{Yb} \\
\text { Concentration }\end{array}$ & $34 \times 10^{25} \mathrm{~m}^{-3}$ & Fitted & \\
\hline$\sigma_{\mathrm{p}, \mathrm{a}}^{\mathrm{Yb}}, \sigma_{\mathrm{p}, \mathrm{e}}^{\mathrm{Yb}}$ & Fig. 7 & Measured & {$[30],[31],[32]$} \\
\hline Yb ${ }^{2} F_{5 / 2}$ life-time & $2 \mathrm{~ms}$ & Assumed & [32] \\
\hline $\begin{array}{l}\mathrm{Yb}{ }^{2} \mathrm{~F}_{5 / 2} \text { quenched } \\
\text { life time }\end{array}$ & $1 \mu \mathrm{s}$ & Assumed & {$[10],[27]$} \\
\hline $\begin{array}{l}\text { Quenched Yb / } \\
\text { Total Yb }\end{array}$ & 0.11 & Measured & \\
\hline Signal Wavelength & $1551-1554 \mathrm{~nm}$ & Measured & \\
\hline $\begin{array}{l}\text { Er } \\
\text { Concentration }\end{array}$ & $3 \times 10^{25} \mathrm{~m}^{-3}$ & Fitted & \\
\hline$\sigma_{\mathrm{s}, \mathrm{a}}^{\mathrm{Er}}, \sigma_{\mathrm{s}, \mathrm{e}}^{\mathrm{Er}}$ & Fig. 8 & Measured & {$[33],[34],[35]$} \\
\hline$\sigma_{p, a}^{\mathrm{Er}}$ & $3 \times 10^{-25} \mathrm{~m}^{2}$ & Assumed & {$[36]$} \\
\hline Er ${ }^{2} \mathrm{I}_{13 / 2}$ life-time & $10 \mathrm{~ms}$ & Assumed & {$[36],[37]$} \\
\hline $\begin{array}{l}\mathbf{E r}{ }^{2} \mathbf{I}_{11 / 2} \text { life-time } \\
\mathrm{Er}^{4} \mathrm{~F}_{7 / 2},{ }^{2} \mathrm{H}_{11 / 2},{ }^{2} \mathrm{~S}_{3 / 2} \\
\text { Non-radiative, } \\
\text { radiative transition } \\
\text { rate }\end{array}$ & $\begin{array}{l}\mathbf{0 . 6} \boldsymbol{\mu S} \\
14 \times 10^{5} \mathrm{~s}^{-1} \\
10^{3} \mathrm{~s}^{-1}\end{array}$ & $\begin{array}{l}\text { Fitted } \\
\text { Assumed }\end{array}$ & $\begin{array}{l}{[23],[38]} \\
{[23]}\end{array}$ \\
\hline $\mathbf{k}_{\mathrm{tr}}$ & $8 \times 10^{-22} \mathrm{~m}^{3} \mathrm{~s}^{-1}$ & Fitted & $\begin{array}{l}{[39],[40],[41],} \\
{[42],[19]}\end{array}$ \\
\hline $\mathrm{C}_{\mathrm{up}, 2}, \mathrm{C}_{\mathrm{up}, 3}$ & $1 \times 10^{-24} \mathrm{~m}^{3} \mathrm{~s}^{-1}$ & Assumed & {$[39],[41]$} \\
\hline $\mathrm{C}_{\mathrm{ESA}, 2, \mathrm{C}}, \mathrm{C}_{\mathrm{ESA}, 3}$ & $1 \times 10^{-27} \mathrm{~m}^{2}$ & Fitted & [43] \\
\hline $\begin{array}{l}\text { Signal Background } \\
\text { Loss }\end{array}$ & $0.15 \mathrm{~m}^{-1}$ & Measured & \\
\hline Core radius & $2.3 \mu \mathrm{m}$ & Measured & \\
\hline $\mathrm{n}_{1}, \mathrm{n}_{2}$ & $1.4709,1.4585$ & Measured & \\
\hline
\end{tabular}

measured this background loss as $0.15 \mathrm{~m}^{-1}(0.65 \mathrm{~dB} / \mathrm{m})$. Again we used McCumber theory in order to calculate the emission cross section assuming emission and absorption cross sections are equal at $1530 \mathrm{~nm}$ [33]. Emission cross section is plotted in Fig. 8(b) with a dotted curve. Resulting spectra are comparable to previously reported spectra [33]-[35].

\section{FITTING PARAMETERS}

The measurements described so far give the emission and absorption cross sections and the percentage of the quenched ions with the assumption of ion concentrations. We either assume a constant value or fit the remaining parameters by comparing the experimental and simulation results. We varied the unknown parameters within the known range until satisfactory fitting is achieved in output powers as well as in residual pump powers for input powers from 0 to $240 \mathrm{~mW}$. Table II shows the fitted, experimentally measured, and assumed parameters with the corresponding literature. References [36]-[43] are additional references for the active medium parameters.

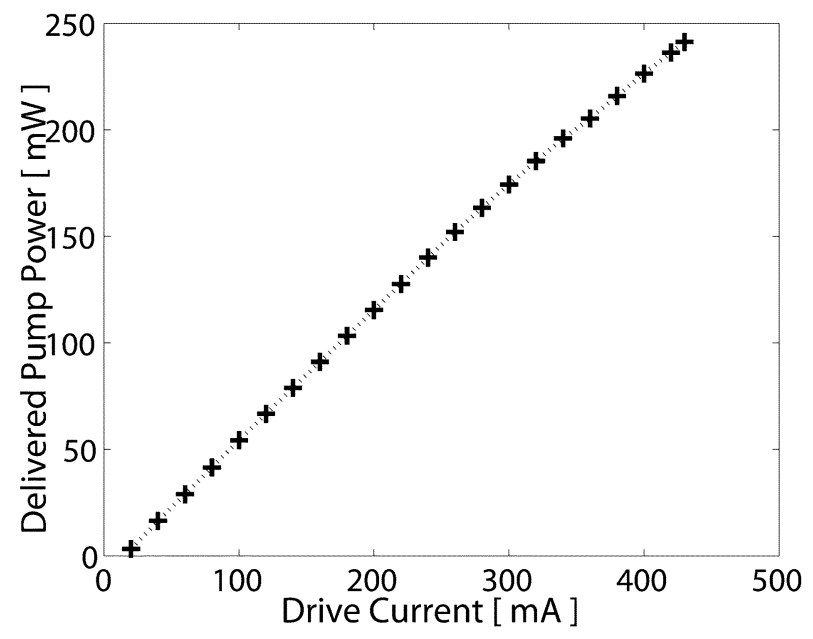

Fig. 9. Drive current versus pump power delivered to the DFB laser after the connectors and splices.

\section{Characterization of the Pump Source}

The semiconductor pump laser we used is electrically pumped with drive current-power characteristics as in Fig. 9. These values are the actual powers delivered to the DFB laser after the wavelength-division-multiplexed (WDM) component.

The wavelength of the pump source is also a defining factor in simulations since it affects the absorption by the active medium. It is especially critical if the pump operates between 970 and 980 $\mathrm{nm}$, where the $\mathrm{Yb}$ absorption cross section dramatically changes with wavelength. As shown in Fig. 10(a), the output spectrum of the pump drifts to longer wavelengths with increasing drive current. Over the tuning range from 50 to $450 \mathrm{~mA}$, the spectrum peak shifts from 978 up to $980 \mathrm{~nm}$. We included this drift in our simulations as a linear variation in the wavelength, as shown in Fig. 10(b).

If the active medium and pump model parameters are accurate enough, then the simulations should predict the absorption characteristics of the fiber without any grating present. The propagation of the pump power $P_{p}$ is governed by (9). In our simulations, we solve this equation by calculating the loss (or gain) term $\alpha_{p}$ for very short segments $(0.2 \mathrm{~mm}$ long) over which we assume the power is constant. Fig. 11(a) shows the simulation (solid curve) and experimental results (error bars) of the cutback experiment at the pump wavelength. Fig. 11(b) compares the theoretical absorption saturation (solid line) with the experimental data (error bars) of the cutback experiment. In the same plot, we also included the simulation results when a constant average pump wavelength ( $979 \mathrm{~nm}$ ) is used (dotted curve) instead of taking into account the drift in the pump wavelength with increasing power, as shown in Fig. 10(b). The deviation between two curves shows the significance of accurate modeling of the pump source, especially at the wavelengths where cross sections change dramatically.

\section{Characterization of the Grating}

The lasing action in a DFB laser is a result of two combined phenomena: signal generation by the active medium and feedback by the grating. Therefore, in addition to the active medium 

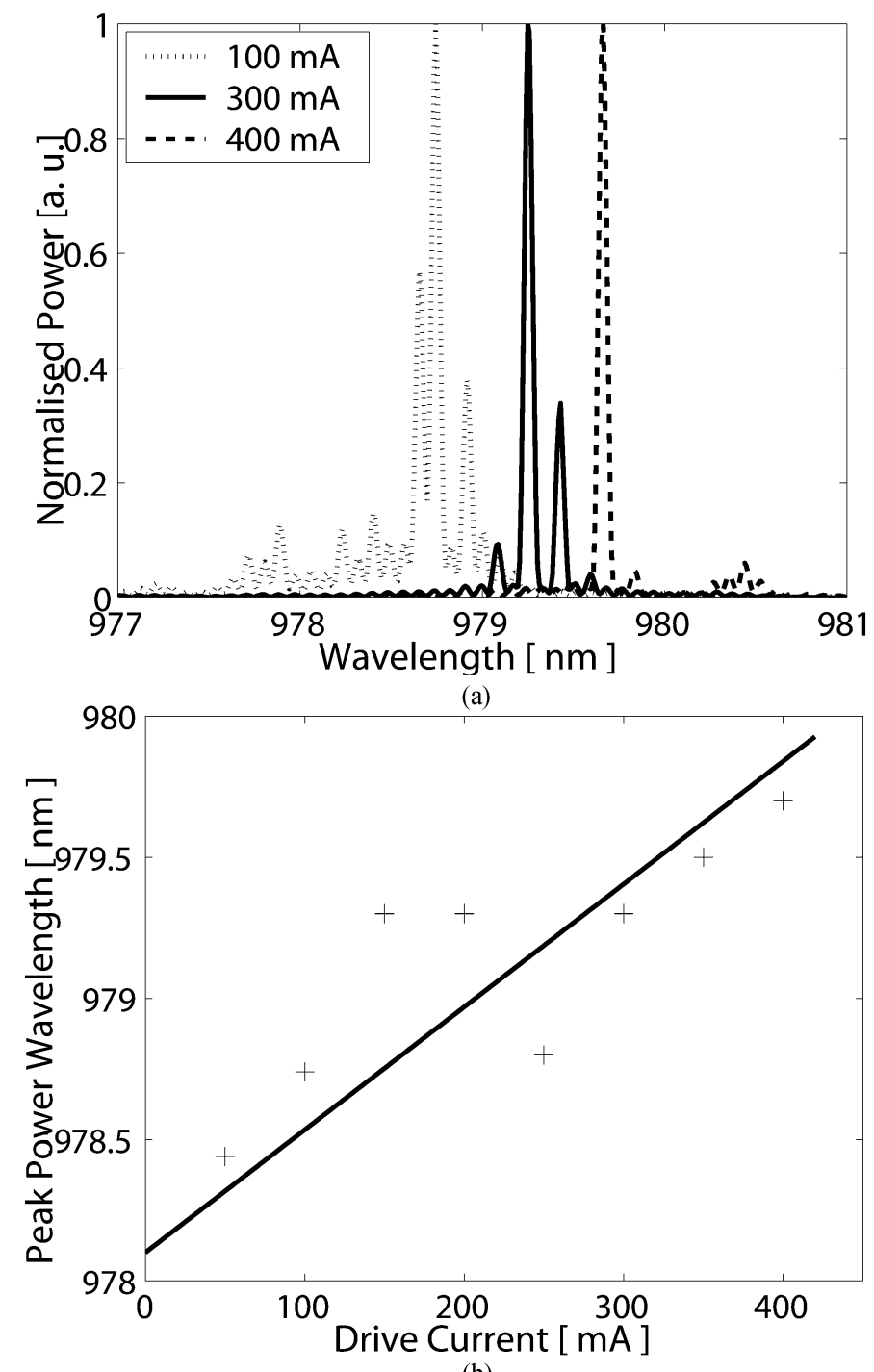

(b)

Fig. 10. (a) Output spectrum of the pump diode for different drive currents. (b) Experimental data $(+)$ of the peak pump power wavelength and linear approximation (solid line) used in the simulations.

parameters, the realistic simulation of a DFB laser requires information about the grating. We acquire this information by analyzing the reflection spectrum of the DFB laser under investigation.

Grating writing procedure is based on a moving translation stage and a stationary phased mask [25], [26]. In this setup, a fiber with a photosensitive ring surrounding the active medium is attached to the translation stage, and a section of grating is written by illuminating the fiber with the UV interference pattern generated by the mask for a fixed exposure time. Then, the translation stage is moved in such a way that next grating section can be written with the desired phase relation with the previous section. Any desired apodization profile is achieved by precisely controlling constructive or destructive superpositioning of consecutive exposures. Changing the overall exposure time changes the refractive-index modulation depth and therefore scales the apodization profile. The grating writing technique guarantees the shape of the apodization; however, in addition to the exposure time, the absolute value of the scaling varies due to the
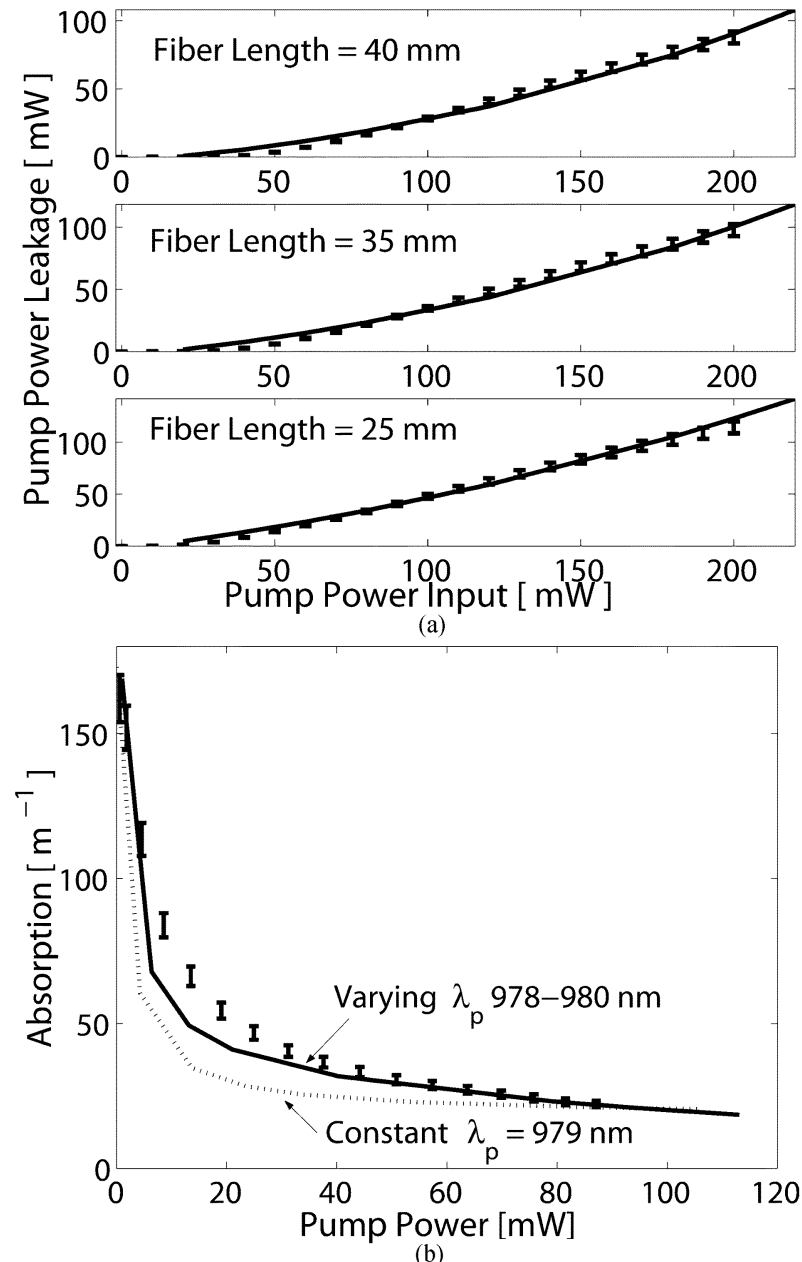

Fig. 11. (a) Comparison of experimental and simulation result of the cutback experiment for 40-, 35-, and 25-mm-long fibers. (b) Variation of average of absorption with pump power. Experimental data (error bars) are compared against the simulation results with varying (solid line) and constant pump wavelength (dotted).

variations in the alignment between the fiber and the UV interference pattern and also due to variations in the photosensitivity of the fiber.

Fig. 12(a) illustrates an apodization profile with three different scales that can be written due to different UV powers delivered or variations in the photosensitivity. The sign change in the apodization profile is equivalent to a $\pi$ shift in the phase of the grating. This shift is introduced by intentionally washing out a $\Lambda$ /4-long grating section, where $\Lambda$ is the period of the grating.

Characterization of the grating corresponds to the measurement of the absolute value of the apodization profile, which manifests itself in the reflection spectrum in a predictable manner: Larger coupling coefficient profile results in larger and wider sidelobes, and a weaker profile leads to smaller and narrower sidelobes in the spectrum of a $\pi$-phase-shifted grating. Fig. 12(b) compares the reflection spectra of the scaled apodization profiles of Fig. 12(a).

When characterizing a DFB laser, since we know the normalized apodization profile from the grating writing process, we can scale it up and down in our simulations until the results match the experimental reflection spectrum data so that we can determine the absolute value of the profile. Although this idea 


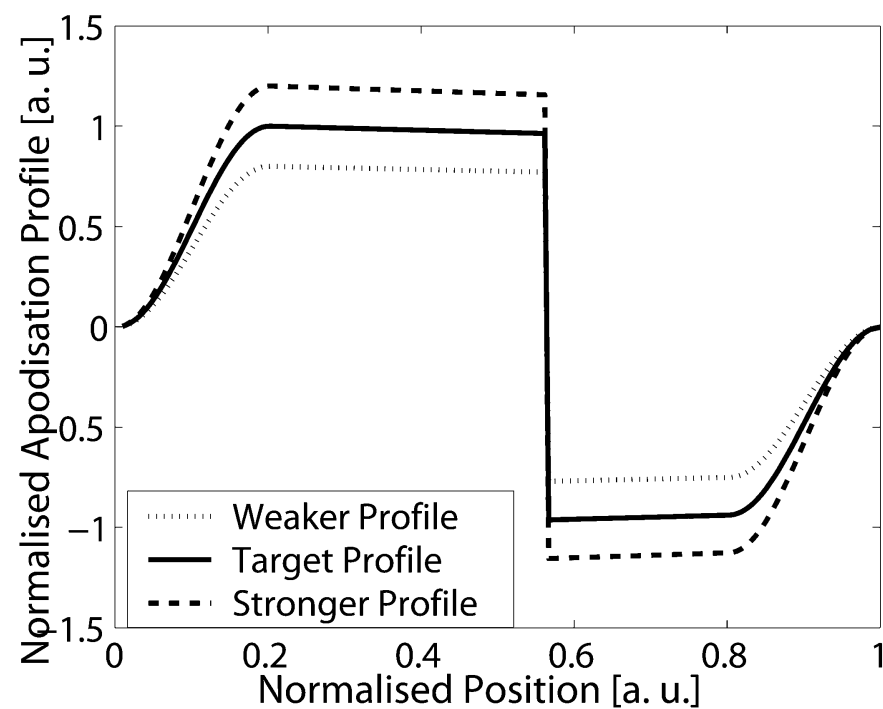

(a)

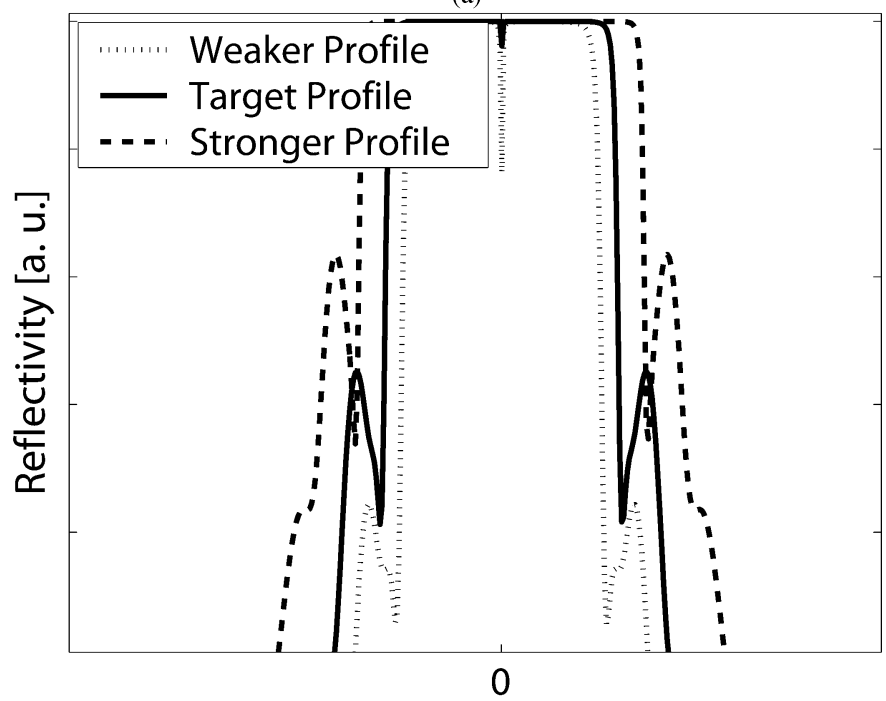

\section{Deviation from the Bragg Wavelength [a. u.]}

(b)

Fig. 12. (a) Normalized apodization profile (solid line) and scaled profiles. Change of sign in the profile corresponds to the discreet $\pi$ phase shift. (b) Normalized reflection spectrum of corresponding apodization profiles.

is simple, in practice the birefringence of the fiber adds some complexity to the measurement. Due to birefringence, fiber has slightly different effective refractive indexes in two orthogonal polarizations. This difference results in two almost identical gratings in two polarizations with a slight offset, which in our case, is $10-20 \mathrm{pm}$. If the measurement setup does not distinguish between these two, then the resultant spectrum will be superimposition of two spectra. Initially, we were not able to separate the two polarizations in our experiments; therefore, we assumed 15-pm offset in our simulations and compared the resultant spectrum to the experimental data. At a later stage, we were able to improve the experimental setup by introducing polarization resolving units, and we compared the spectra for individual polarizations.

We used a tunable laser source (TLS) in order to measure the reflection spectra of DFB lasers. Fig. 13 shows the TLS output spectrum across the tuning range. Since the power delivered to the grating is not constant, we also incorporated this variation

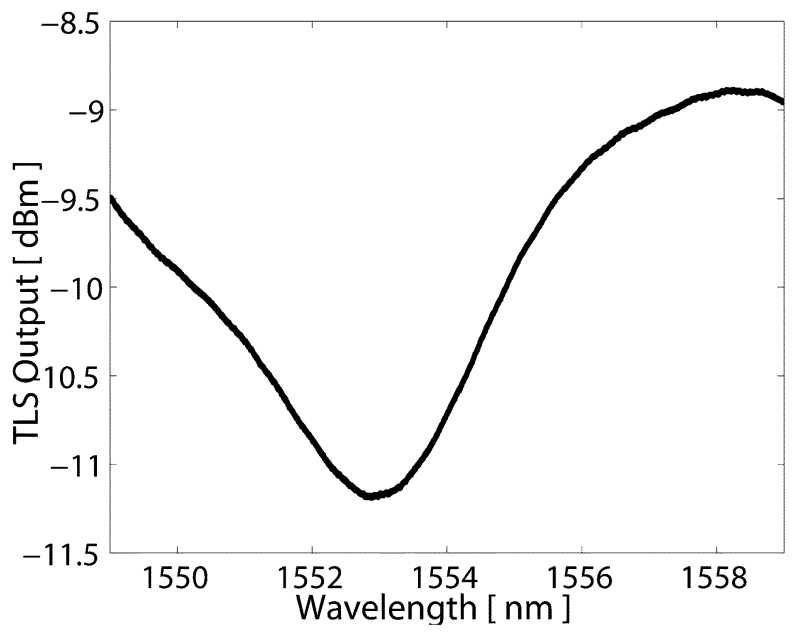

Fig. 13. Power output spectrum of tunable laser source used for grating characterization. The variation in the power manifests itself as a slope in the reflection spectrum.

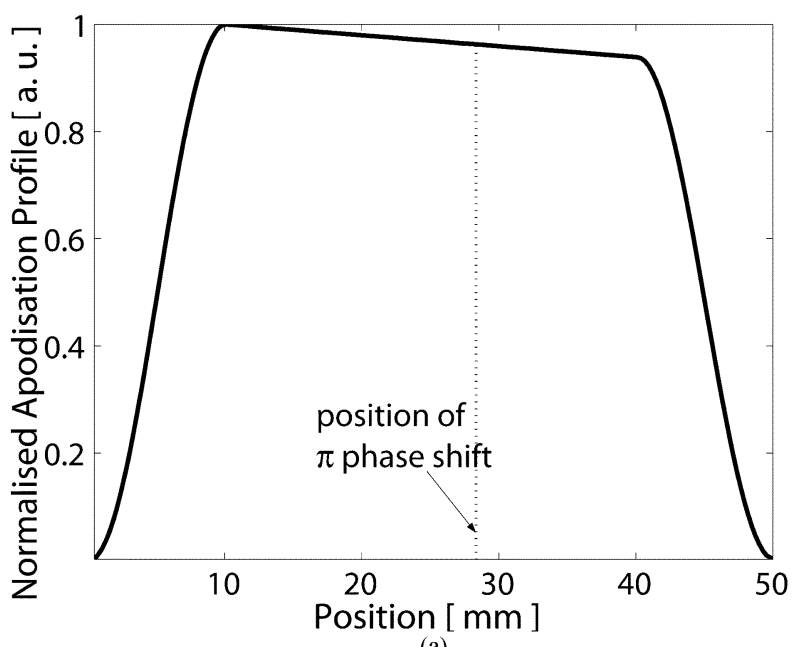

(a)

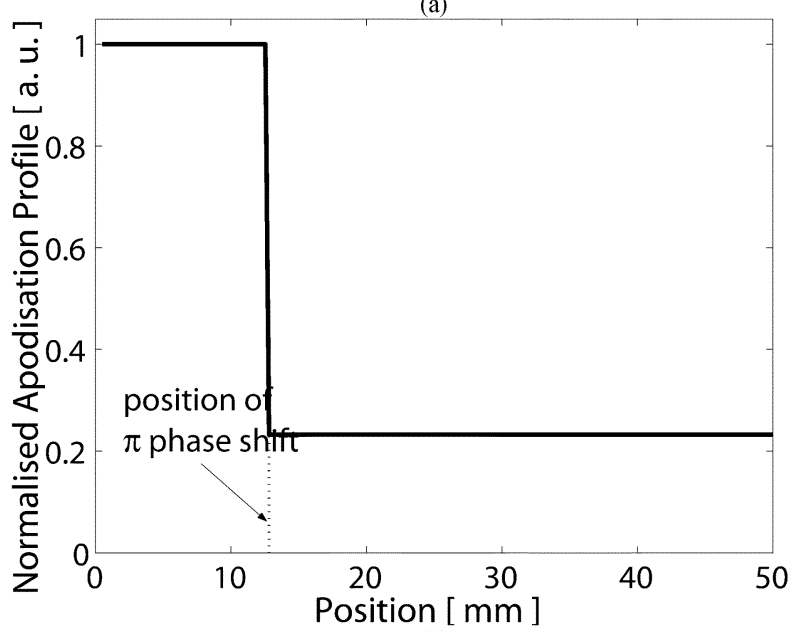

(b)

Fig. 14. Two normalized apodization profiles used in DFB lasers. (a) Smooth apodized profile. (b) Step apodized profile.

in the incident power with a wavelength when calculating the reflection in our simulations.

Fig. 14(a) and (b) show two different normalized apodization profiles, used in DFB lasers, in absolute scale, where the position of the phase shift is indicated with the dotted vertical lines. 

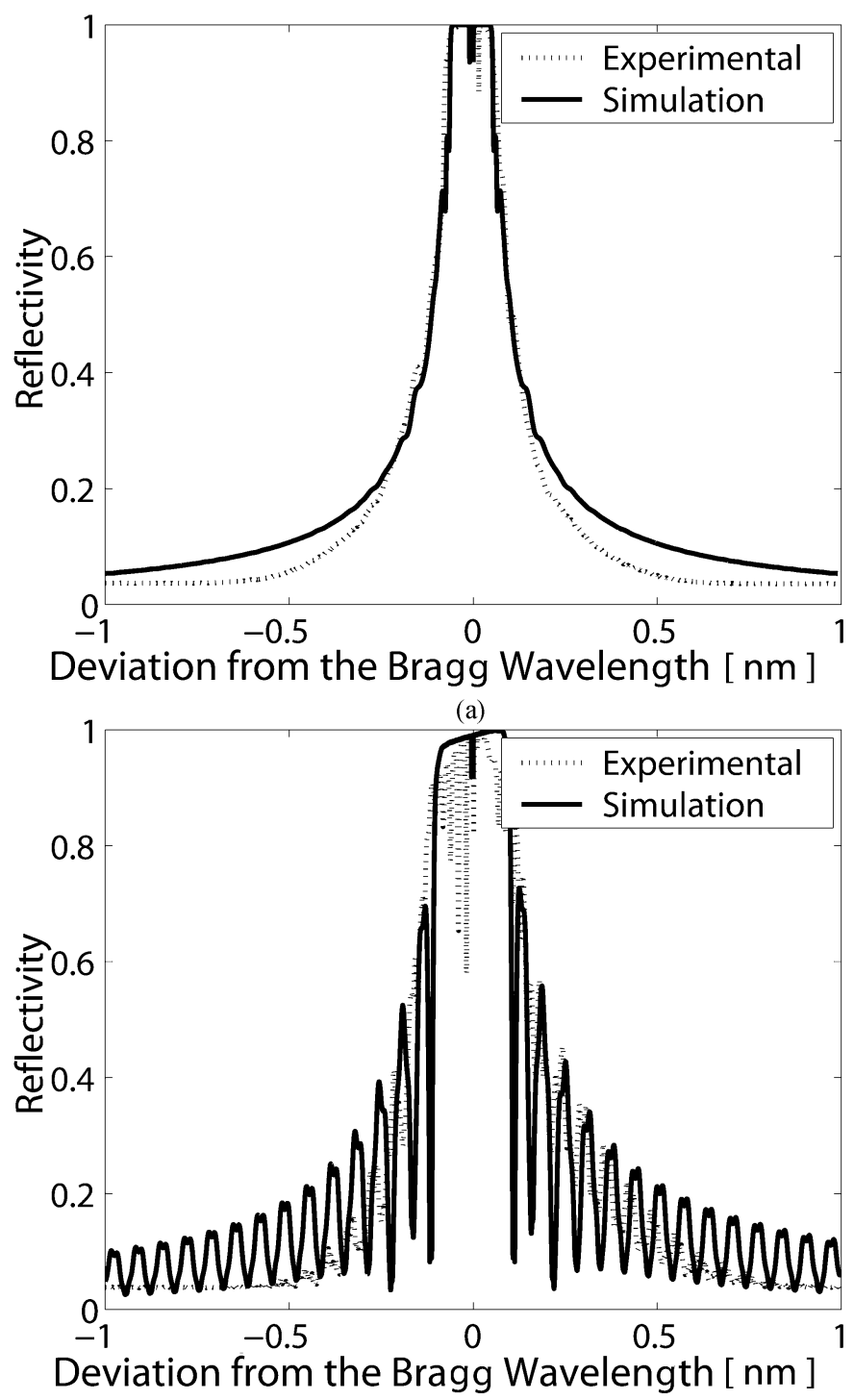

(b)

Fig. 15. Deduction of absolute scaling of the apodization profiles by comparing the experimental (dotted line) and theoretical (solid line) reflection spectra. (a) Smooth apodized profile. Peak $\kappa=210 \mathrm{~m}^{-1}$. (b) Step apodized profile. Peak $\kappa=350 \mathrm{~m}^{-1}$.

Fig. 14(a) is a standard DFB laser. The small apodization toward the ends increases the side-mode suppression and improves the longitudinal mode stability. The asymmetrically located phase shift provides unidirectional output power. The step apodized profile given in Fig. 14(b) is discussed in detail somewhere else [44]. It is designed to improve the efficiency, and it still maintains the same unidirectionality and longitudinal mode stability. The normalized profiles are inherently known from the production method, and we used the grating characterization method to find the scaling factor, which is equivalent to the grating's maximum coupling coefficient $\kappa$. Fig. 15(a) and (b) shows the matching simulation and experimental reflection spectra (Bragg wavelength $=1552 \mathrm{~nm}$ ) for apodization profiles in Fig. 14(a) and (b), respectively, including 15-pm birefringence. We found the scaling factor to be $210 \mathrm{~m}^{-1}$ and $350 \mathrm{~m}^{-1}$ in the respective cases.

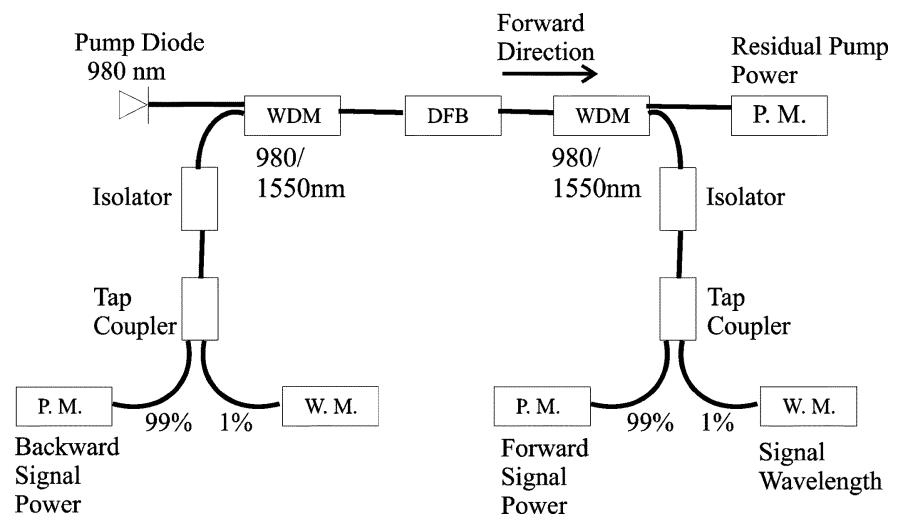

Fig. 16. Experimental setup used to characterize DFB lasers.

Experimental and simulations results for reflection spectra are both composed of two slightly offset spectra due to birefringence and simulations take into account the wavelength dependent nature of the TLS output shown in Fig. 13. The difference between the experimental and simulation reflected powers found to be less than $-32 \mathrm{dBm}(0.6 \mu \mathrm{W})$ for input powers around $-11 \mathrm{dBm}$.

\section{DFB LASER SIMULATION AND EXPERIMENT}

After characterizing the pump source, deducing the absolute apodization profile of the gratings and confirming the active medium model predictions at both high and low pump powers, we are now ready to simulate DFB lasers and compare with the experimental results. The simulation of DFB lasers are carried out by using the transfer matrix method [45]. In this method, a nonuniform laser structure is divided into short segments over which the fiber and cavity properties as well as the propagating fields are assumed to be constant. Each segment is characterized by a transfer matrix that relates the fields on one side to the fields on the other. The overall laser characteristics are obtained by multiplying the individual transfer matrices sequentially.

Fig. 16 shows the experimental setup used to characterize the DFB lasers. DFB lasers are pumped by the semiconductor laser in a codirectional manner: that is, the pump power propagates in the same direction as the desired output end of the DFB laser. In this forward direction, a 980/1550-nm WDM is used to separate the DFB output signal from the residual pump power. Residual pump power is measured by a power meter (PM).

A tap coupler is used to feed $1 \%$ of the signal to a wave meter (WM) in order to measure the peak wavelength. The other arm of the coupler is connected to a PM to measure the output power in the forward direction. A similar setup is used on the other side of the DFB laser in order to measure the backward output power as well as the peak wavelength. On both sides, optical isolators prevent spurious back-reflections from entering and perturbing the DFB laser. The transmission of all components is measured so that the output powers could be calculated from PM readings.

The theoretical and experimental output power at $1552 \mathrm{~nm}$ and the residual pump power around $979 \mathrm{~nm}$ from the two DFB designs given in Fig. 14(a) and (b) are shown in Fig. 17(a) and (b), respectively. The results for both designs show very good agreement over the full range of the pump powers.

The deviation between simulation and experimental results at low pump powers, around threshold, can be attributed to 


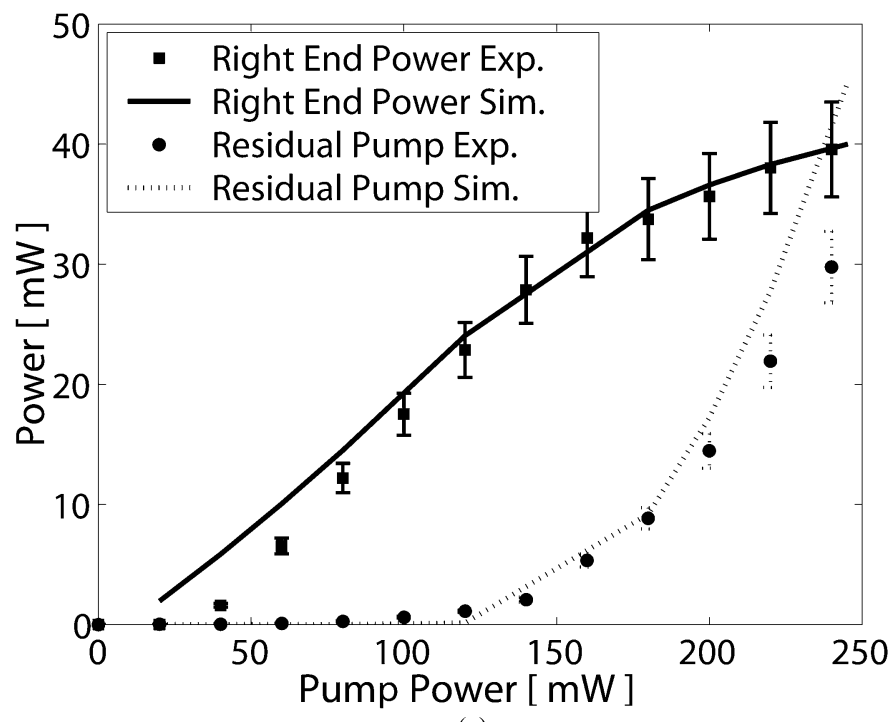

(a)

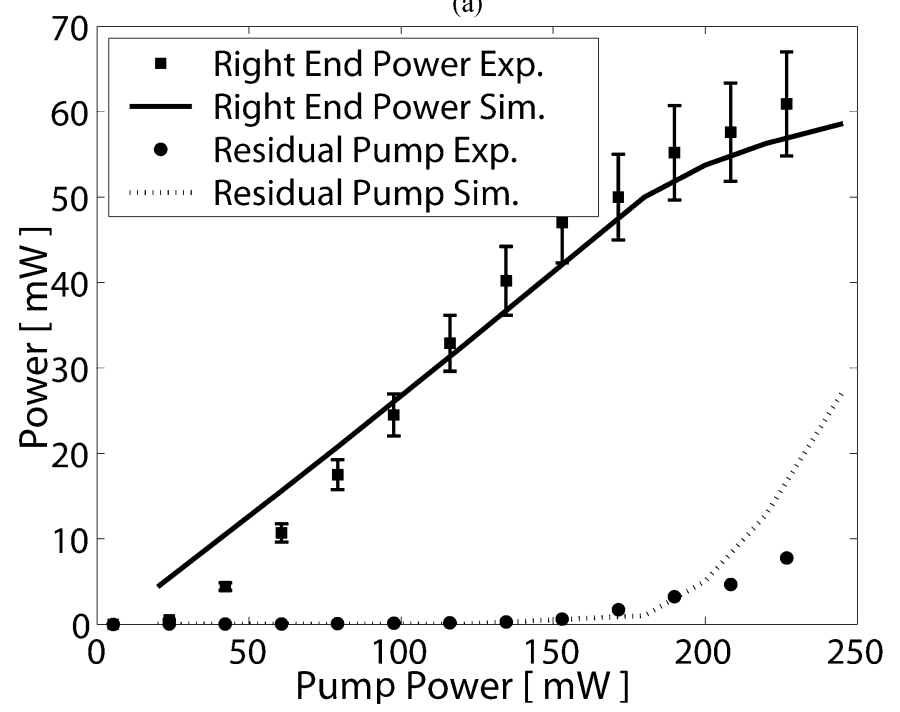

(b)

Fig. 17. Design-end signal and residual pump power from DFB lasers. Experimental (error bars) and theoretical data (solid and dotted lines) are compared. (a) Smooth apodized DFB laser. (b) Step apodized DFB laser.

a number of factors: the initial phase shift could be slightly different from the ideal $\pi$ value, which would result in larger threshold power and smaller efficiency than the ideal case, as observed at the low power end of Fig. 17(a) and (b). However, differential heat dissipation and nonuniform temperature distributions are known to affect the gratings in Er-Yb-co-doped fibers [46]. At the larger pump powers, as a result of heating, the phase shift could be approaching $\pi$, leading to an improved agreement between experiment and theory. In addition, unaccounted coupling-constant variations along the grating length can also contribute to threshold discrepancies. Finally, uncertainties related with the exact pump-wavelength variations with pump power [see Fig. 10(b)], and the exact shape of the absorption peak around $979 \mathrm{~nm}$ can also contribute further to the observed discrepancies.

\section{CONCLUSION}

This paper describes how Er-Yb-co-doped $\pi$-phase-shifted fiber DFB lasers were modeled as complete systems comprised of the pump source, the active medium, and the grating. Each of them were characterized and, for the first time, two different DFB laser designs were simulated with experimental verification over a pump power range from 0 to $240 \mathrm{~mW}$. Unlike previous theoretical works, which gave information as trends in the power characteristics, the results agreed well with the actual experimental data for different DFB laser designs. The agreement was quite satisfactory at large output powers where the lasers are intended to be used. The matching at low powers could be improved further by using a grating stabilized pump source in order to prevent uncontrolled variations in the pump wavelength, as well as by better defining the actual grating phase shift and measuring more accurately the losses and errors introduced by cleaving and splicing.

A set of simple experiments were presented for the characterization of Er-Yb-doped media and gratings that provide sufficient data for the modeling and simulation of short fibers and lasers. A large amount of unsaturable loss at the pump wavelength that is attributed to $\mathrm{Yb}$ clustering and lifetime quenching was measured. This effect has a significant impact on the performance and the efficiency of fiber DFB lasers. This proves to be the major limiting factor in the design of highly efficient DFB lasers [47], [48].

The developed model can be utilized in many different ways: it can be used to compare laser designs with different apodization profiles, lengths, and chirp rates, in terms of their efficiency at different pump wavelengths and pump powers. It can also be used to investigate the effects of the fiber geometry such as core radius, or numerical aperture (NA), the active medium properties, such as quenching, or ion concentration on the device performance at different signal wavelengths.

\section{ACKNOWLEDGMENT}

Distributed feedback laser production and characterization were carried out at the state-of-art facilities of SPI (Southampton Photonics, Inc.), Southampton, U.K. The authors would like to thank the SPI manufacturing staff for their help in the experimental verification of the theory.

\section{REFERENCES}

[1] J. Hubner, P. Varming, and M. Kristensen, "Five wavelength DFB fiber laser source for WDM systems," Electron. Lett., vol. 33, no. 2, pp. 139-140, 1997.

[2] M. Ibsen, S. U. Alam, M. N. Zervas, A. B. Grudinin, and D. N. Payne, "8-and 16-channel all-fiber DFB laser WDM transmitters with integrated pump redundancy," IEEE Photon. Technol. Lett., vol. 11, no. 9, pp. 1114-1116, Sep. 1999.

[3] H. N. Poulsen, P. Varming, A. Buxens, A. T. Clausen, P. Munoz, P. Jeppesen, C. V. Poulsen, J. E. Pedersen, and L. Eskildsen, "1607 nm DFB fiber laser for optical communcation in the L-band," presented at the Eur. Conf. Optical Communications (ECOC), Nice, France, 1999.

[4] J. T. Kringlebotn, W. H. Loh, and R. I. Laming, "Polarimetric $\mathrm{Er}^{3+}$-doped fiber distributed-feedback laser sensor for differential pressure and force measurements," Opt. Lett., vol. 21, no. 22, pp. $1869-1871,1996$

[5] E. Ronnekleiv, M. Ibsen, and G. J. Cowle, "Polarization characteristics of fiber DFB lasers related to sensing applications," IEEE J. Quantum Electron., vol. 36, no. 6, pp. 656-664, Jun. 2000.

[6] O. Hadeler, M. Ibsen, and M. N. Zervas, "Distributed-feedback fiber laser sensor for simultaneous strain and temperature measurements operating in the radio-frequency domain," Appl. Opt., vol. 40, no. 19, pp. 3169-3175, 2001.

[7] E. Ronnekleiv, "Frequency and intensity noise of single frequency fiber Bragg grating lasers," Opt. Fiber Technol., vol. 7, no. 3, pp. 206-235, 2001. 
[8] L. B. Fu, R. Selvas, M. Ibsen, J. K. Sahu, J. N. Jang, S. U. Alam, J. Nilsson, D. J. Richardson, D. N. Payne, C. Codemard, S. Goncharov, I. Zalevsky, and A. B. Grudinin, "Fiber-DFB laser array pumped with a single 1-W CW Yb-fiber laser," IEEE Photon. Technol. Lett., vol. 15, no. 5, pp. 655-657, May 2003.

[9] C. Strohhofer and A. Polman, "Relationship between gain and $\mathrm{Yb}^{3+}$ concentration in $\mathrm{Er}^{3+}-\mathrm{Yb}^{3+}$ doped waveguide amplifiers," J. Appl. Phys., vol. 90, no. 9, pp. 4314-4320, 2001.

[10] R. Paschotta, J. Nilsson, P. R. Barber, J. E. Caplen, A. C. Tropper, and D. C. Hanna, "Lifetime quenching in Yb-doped fibers," Optics Commun., vol. 136375-136378, 1997.

[11] S. B. Poole, J. E. Townsend, D. N. Payne, M. E. Fermann, G. J. Cowle, R. I. Laming, and P. R. Morkel, "Characterization of special fibers and fiber devices," J. Lightw. Technol., vol. 7, no. 8, pp. 1242-1255, Aug. 1989

[12] G. G. Vienne, J. E. Caplen, L. Dong, J. D. Minelly, J. Nilsson, and D. N. Payne, "Fabrication and characterization of $\mathrm{Yb}^{3+}: \mathrm{Er}^{3+}$ phosphosilicate fibers for laser," J. Lightw. Technol., vol. 16, no. 11, pp. 1990-2001, Nov. 1998

[13] G. Sorbello, S. Taccheo, and P. Laporta, "Numerical modeling and experimental investigation of double-cladding erbium-ytterbium-doped fiber amplifiers," Optical Quantum Electron., vol. 33 599-33 619, 2001.

[14] E. Yahel and A. Hardy, "Modeling high-power $\mathrm{Er}^{3+}-\mathrm{Yb}^{3+}$ codoped fiber lasers," J. Lightw. Technol., vol. 21, no. 9, pp. 2044-2052, Sep. 2003

[15] - "Modeling and optimization of short $\mathrm{Er}^{3+}-\mathrm{Yb}^{3+}$ codoped fiber lasers," IEEE J. Quantum Electron., vol. 39, no. 11, pp. 1444-1451, Nov. 2003

[16] M. Karasek, "Optimum design of $\mathrm{Er}^{3+}{ }^{3} \mathrm{Yb}^{3+}$ codoped fibers for largesignal high-pump-power applications," IEEE J. Quantum Electron., vol. 33, no. 10, pp. 1699-1705, Oct. 1997.

[17] G. C. Valley, "Modeling cladding-pumped Er/Yb fiber amplifiers," $O p$ tical Fiber Technol., vol. 721-44, 2001.

[18] F. Di Pasquale, "Modeling of highly-efficient grating-feedback and Fabry-Pérot $\mathrm{Er}^{3+} \mathrm{Yb}^{3+}$ co-doped fiber lasers," IEEE J. Quantum Electron., vol. 32, no. 2, pp. 326-332, Feb. 1996.

[19] J. Nilsson, P. Scheer, and B. Jaskorzynska, "Modeling and optimization of short $\mathrm{Yb}^{3+}$ sensitized $\mathrm{Er}^{3+}$ doped fiber amplifiers," IEEE Photon. Technol. Lett., vol. 6, no. 3, pp. 383-385, Mar. 1994.

[20] M. Achtenhagen, R. J. Beeson, F. Pan, B. Nyman, and A. Hardy, "Gain and noise in ytterbium-sensitized erbium-doped fiber amplifiers: Measurements and simulations," J. Lightw. Technol., vol. 19, no. 10, pp. 1521-1526, Oct. 2001

[21] J. Kemtchou, M. Duhamel, and P. Lecoy, "Gain temperature dependence of erbium-doped silica and fluoride fiber amplifiers in multichannel wavelength-multiplexed transmission systems," J. Lightw. Technol., vol. 15, no. 11, pp. 2083-2090, Nov. 1997.

[22] Y. Maeda and T. Yamada, "Temperature dependence of the enhanced excited state absorption in erbium-doped garnets," J. Appl. Phys., vol. 83, no. 12, pp. 7436-7441, 1998.

[23] E. Desurvire, C. R. Giles, and J. R. Simpson, "Gain saturation effects in high-speed, multichannel Erbium-doped fiber amplifiers at Lambda =

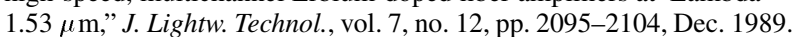

[24] L. Dong, W. H. Loh, J. E. Caplen, J. D. Minelly, K. Hsu, and L. Reekie, "Efficient single-frequency fiber lasers with novel photosensitive $\mathrm{Er} / \mathrm{Yb}$ optical fibers," Opt. Lett., vol. 22, no. 10, pp. 694-696, 1997.

[25] W. H. Loh, M. J. Cole, M. N. Zervas, S. Barcelos, and R. I. Laming, "Complex grating structures with uniform phase masks based on the moving fiber-scanning beam technique," Opt. Lett., vol. 20, no. 20, pp. 2051-2053, 1995.

[26] A. Asseh, H. Storoy, B. E. Sahlgren, S. Sandgren, and R. A. H. Stubbe, "A writing technique for long fiber Bragg gratings with complex reflectivity profiles," J. Lightw. Technol., vol. 15, no. 8, pp. 1419-1423, Aug. 1997

[27] Z. Burshtein, Y. Kalisky, S. Z. Levy, P. Le Boulanger, and S. Rotman, "Impurity local phonon nonradiative quenching of $\mathrm{Yb}^{3+}$ fluorescence in ytterbium-doped silicate glasses," IEEE J. Quantum Electron., vol. 36, no. 8, pp. 1000-1007, Aug. 2000.

[28] P. Yang, P. Deng, and Z. Yin, "Concentration quenching in Yb:YAG," $J$. Lumin., no. 97, pp. 51-52, 2002.

[29] E. Desurvire, "Section 4.5 determination of transition cross sections," in Erbium-Doped Fiber Amplifiers: Principles and Applications. New York: Wiley, 1994, pp. 245-270.

[30] H. M. Pask, R. J. Carman, D. C. Hanna, A. C. Tropper, C. J. Mackechnie, P. R. Barber, and J. M. Dawes, "Ytterbium-doped silica fiber lasers: Versatile sources for the 1-1.2 $\mu \mathrm{m}$ region," IEEE J. Select. Topics Quantum Electron., vol. 1, no. 1, pp. 2-13, Apr. 1995.
[31] X. Zou and H. Toratani, "Evaluation of spectroscopic properties of $\mathrm{Yb}^{3+}$-doped glasses," Phys. Rev. B, Condens. Matter, vol. 52, no. 22, pp. $15889-15897,1995$.

[32] H. Yin, P. Deng, J. Zhang, and F. Gan, "Emission properties of $\mathrm{Yb}^{3+}$ in fluorophosphate glass," J. Non-Cryst. Solids, vol. 210 243-210248, 1997.

[33] W. J. Miniscalco and R. S. Quimby, "General procedure for the analysis of Er ${ }^{3+}$ cross sections," Opt. Lett., vol. 16, no. 4, pp. 258-260, 1991.

[34] W. J. Miniscalco, "Erbium-doped glasses for fiber amplifiers at 1500 nm," J. Lightw. Technol., vol. 9, no. 2, pp. 234-250, Feb. 1991.

[35] L. W. Barnes, R. I. Laming, E. J. Tarbox, and P. R. Morkel, "Absorption and emission cross section of $\mathrm{Er}^{3+}$ doped silica fibers," IEEE $J$. Quantum Electron., vol. 27, no. 4, pp. 1004-1010, Apr. 1991.

[36] E. Desurvire, J. L. Zyskind, and C. R. Giles, "Design optimization for efficient erbium-doped fiber amplifiers," J. Lightw. Technol., vol. 8, no. 11, pp. 1730-1741, Nov. 1990.

[37] B. J. Ainslie, "A review of the fabrication and properties of erbiumdoped fibers for optical amplifiers," J. Lightw. Technol., vol. 9, no. 2, pp. 220-227, Feb. 1991.

[38] E. Maurice, G. Monnom, B. Dussardier, and D. B. Ostrowsky, "Clustering effects on double energy transfer in heavily ytterbium-erbiumcodoped silica fibers," J. Opt. Soc. Amer. B, Opt. Phys., vol. 13, no. 4 pp. 693-701, 1996.

[39] B. Hwang, S. Jiang, T. Luo, J. Watson, G. Sorbello, and N. Peyghambarian, "Cooperative upconversion and energy transfer of new high $\mathrm{Er}^{3+}$ - and $\mathrm{Yb}^{3+}-\mathrm{Er}^{3+}{ }_{-}$doped phosphate glasses," J. Opt. Soc. Amer. B, Opt. Phys., vol. 17, no. 5, pp. 833-838, 2000.

[40] C. Lester, A. Bjarklev, T. Rasmussen, and P. G. Dinesen, "Modeling of $\mathrm{Yb}^{3+}$ sensitized $\mathrm{Er}^{3+}$ doped silica waveguide amplifiers," J. Lightw. Technol., vol. 13, no. 5, pp. 740-743, May 1995.

[41] S. Taccheo, G. Sorbello, S. Longhi, and P. Laporta, "Measurement of the energy transfer and upconversion constants in Er-Yb-doped phosphate glass," Optical Quantum Electron., vol. 31 249-31 262, 1999.

[42] M. Federighi and F. Di Pasquale, "The effect of pair-induced energy transfer on the performance of Silica waveguide amplifiers with high $\mathrm{Er}^{3+} / \mathrm{Yb}^{3+}$ concentrations," IEEE Photon. Technol. Lett., vol. 7, no. 3 , pp. 303-305, Mar. 1995

[43] J. Nilsson, P. Blixt, B. Jaskorzynska, and J. Babonas, "Evaluation of parasitic upconversion mechanisms in $\mathrm{Er}^{3+}$-doped Silica-glass fibers by analysis of fluorescence at $980 \mathrm{~nm}$, , J. Lightw. Technol., vol. 13, no. 3, pp. 341-349, 1995.

[44] K. Yelen, L. M. B. Hickey, and M. N. Zervas, "A new design approach for fiber DFB lasers with improved efficiency," IEEE J. Quantum Electron., vol. 40, no. 6, pp. 711-720, Jun. 2004.

[45] M. Yamada and K. Sakuda, "Analysis of almost-periodic distributed feedback slab waveguides via a fundamental matrix approach," Appl. Opt., vol. 26, no. 16, pp. 3474-3478, 1987.

[46] Y. Z. Xu, H. Y. Tam, S. Y. Liu, and M. S. Demokan, "Pump-induced thermal effects in Er-Yb fiber grating DBR lasers," IEEE Photon. Technol. Lett., vol. 10, no. 9, pp. 1253-1255, Sep. 1998.

[47] K. Yelen, M. N. Zervas, and L. M. B. Hickey, "Fiber DFB lasers with ultimate efficiency," presented at the Optical Fiber Communications Conf. (OFC), Los Angeles, CA, 2004.

[48] - "Fiber DFB lasers with ultimate efficiency," J. Lightw. Technol., vol. 23 , no. 1, pp. 32-43, Jan. 2005

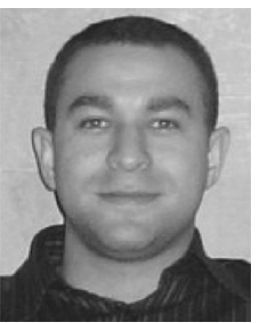

Kuthan Yelen was born in Edirne, Turkey, on June 27, 1977. He received the B.Sc. degree in electrical and electronics engineering from the Middle East Technical University (METU) of Ankara, Ankara, Turkey, in 1999, the M.Sc. degree in optical fiber communications from the Department of the Electronics and Computer Science, University of Southampton, Southampton, U.K., in 2001, and the $\mathrm{Ph} . \mathrm{D}$. degree from the Optoelectronics Research Centre (ORC) of the same university in 2004, where he developed the design method for ultimate efficiency in linear-cavity continuous-wave lasers using distributed feedback.

His research interests include computational photonics, characterization, modeling, simulation, and design of lasers, rare-earth-doped devices, and periodic structures.

Dr. Yelen was the recipient of scholarships from the National Education Foundation (MEV) of Turkey between 1995 and 1999 and from Turkish Education Foundation (TEV) between 1999 and 2000. He was a Chevening Scholar of the British Council during the same period. Between 2001 and 2004, he received a research scholarship from the University of Southampton and the Overseas Research Students Award (ORS) from the Committee of Vice-Chancellors and Principals of the United Kingdom (CVCP). 


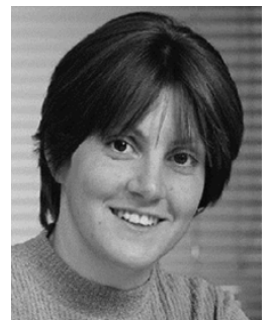

Louise M. B. Hickey received the B.S. degree in physics with laser science from the University of Southampton, Southampton, U.K., in 1993 and the $\mathrm{Ph} . \mathrm{D}$. degree from the Department of Electronics and Computer Science and Optoelectronics Research Centre of the same university, during which time her main field of study was planar waveguides and lasers in Ti-diffused sapphire.

From 1997 to 1999, she was awarded a Fellowship from the Royal Commission of the Exhibition of 1851 to continue her research into planar waveguide lasers at the Optoelectronics Research Centre, Southampton University. Following a year with De Beers Industrial Diamonds, she joined SPI (Southampton Photonics, Inc.), Southampton, U.K., in 2001 and is currently working to extend the power and performance of in-fiber lasers.

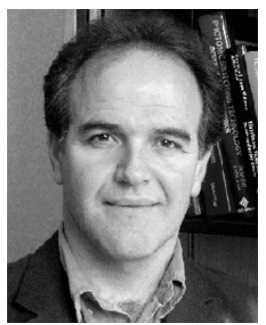

Mikhail N. Zervas ( $\left.\mathrm{S}^{\prime} 88-\mathrm{M}^{\prime} 88\right)$ was born in Dimaina, Nafplion, Greece, on June 1, 1959. He received the Bachelor's degree from the Electrical Engineering Department, University of Thessaloniki, Thessaloniki, Greece, in 1984, the M.Sc. degree in applied and modern optics from the University of Reading, Reading, U.K., in 1985, and the Ph.D. degree in fiber optics from the University College London, London, U.K., in 1989, respectively.

He joined the Optoelectronics Research Centre, University of Southampton, Southampton, U.K., in 1991 as a Research Fellow and was promoted to Research Lecturer in 1995 and Professor in Optical Communications in 1999. His research activities include advanced optical fiber amplifier configurations, high-power fiber lasers, fiber distributed feedback lasers, Bragg grating theory and devices, surface-plasmon effects and devices, optical trapping, and nanoparticle manipulation. He is one of the co-founders of SPI (Southampton Photonics, Inc.), Southampton, U.K., where he is currently Chief Scientist while on a university sabbatical leave. He has authored/coauthored more than 170 technical publications and 25 patents/patent applications.

Dr. Zervas shared with his colleagues the prestigious Metrology Award from the Confederation of British Industry in 1996 for their work on grating measuring systems for characterizing reflection and dispersion performance of fiber Bragg gratings. He has served on various conference program committees, and he was the general program co-chair of the 1999 Optical Amplifiers Meeting (OSA) in Japan. He has given a number of invited talks and short courses on fiber gratings and fiber amplifiers at major international conferences. He was a co-editor of the Integrated and Fiber Optics journal's Special Issue on Fiber Bragg Gratings. 